\title{
Multiple access spatial modulation
}

\author{
Nikola Serafimovski ${ }^{*}$, Sinan Sinanović ${ }^{1}$, Marco Di Renzo ${ }^{2}$ and Harald Haas ${ }^{1}$
}

\begin{abstract}
In this study, we seek to characterise the behaviour of Spatial modulation (SM) in the multiple access scenario. By only activating a single transmit antenna for any transmission, SM entirely avoids inter-channel interference, requires no synchronisation between the transmit antennas and a single radio frequency chain at the transmitter. Most contributions thus far have only addressed aspects of SM for a point-to-point communication system. We propose a maximum-likelihood (ML) detector which can successfully decode incoming data from multiple simultaneous transmissions and does not suffer from the near-far problem. We analyse the performance of the interference-unaware and interference-aware detectors. We look at the behaviour of SM as the signal-to-interference-plus-noise ratio goes to infinity and compare it to the complexity and cost equivalent single-input-multiple-output (SIMO) system. Two systems are considered to be equivalent in terms of complexity if their respective detection algorithms are of the same order in $\mathcal{O}(\cdot)$ notation. Simulation results show that the interference-aware SM detector performs better than the complexity equivalent multi-user ML-SIMO detector by at least $3 \mathrm{~dB}$ at an average bit-error-ratio of $10^{-3}$.
\end{abstract}

\section{Introduction}

Multiple-antenna systems are fast becoming a key technology for modern wireless systems. They offer improved error performance and higher data rates, at the expense of increased complexity and power consumption [1]. Spatial modulation (SM) is a recently proposed approach to multiple-input-multiple-output (MIMO) systems which entirely avoids inter-channel interference, requires no synchronisation between the transmit antennas and achieves a spatial multiplexing gain [2]. This is performed by mapping a block of information bits into a constellation point in the signal and spatial domains [3]. In SM, the number of information bits, $\ell$, that are encoded in the spatial domain can be related to the number of transmit antennas $N_{t}$ as $N_{t}=2^{\ell}$. This means that the number of transmit antennas must be a power of two unless fractional bit encoding [4] or generalised SM [5] are used. SM offers an intrinsic flexibility to trade off the number of transmit antennas with the modulation order in the signal domain to meet the desired data rate. It should be noted that SM is shown to outperform other point-topoint MIMO schemes in terms of average bit-error-ratio (ABER) [3].

\footnotetext{
*Correspondence: n.serafimovski@ed.ac.uk

${ }^{1}$ Institute for Digital Communications, Joint Research Institute for Signal and Image Processing, School of Engineering, The University of Edinburgh, Edinburgh EH9 3JL, UK

Full list of author information is available at the end of the article
}

In the single user scenario, only a single transmit antenna is active at any instance, this avoids the need for complicated interference cancellation algorithms at the SM receiver. In addition, unlike other MIMO schemes, the number of receive antennas is independent of the number of transmit antennas. Several articles are available in literature which are aimed at understanding and improving the performance of SM in various scenarios, e.g., [6-8]. The study in [6] seeks to improve the ABER performance of SM by introducing trellis coding on the transmitting antennas. The study in [9] shows that the detector complexity of SM is independent of the number of transmit antennas. The optimal detector is known with and without channel state information at the receiver in [10-12]. The optimal power allocation problem for a two-transmit with one receive antenna system is solved in closed form in [13] and the performance of SM in correlated fading channels is considered in $[14,15]$. Recent work has also shown that SM can be combined with space-time block codes to attain spectral efficiency gains [16] by exploiting transmit-side diversity. At this point, it is worth noting that if we choose to use only the spatial constellation of SM to transmit information, then SM is reduced to spaceshift-keying (SSK) as proposed in [17]. To this extent, we note that all presented work can be extended to SSK without loss of generality.

MIMO techniques can also be used in relaying networks to improve the diversity, provide multiplexing gains and

\section{Springer}


aid in interference cancellation. To this extent, the orthogonal decode and forward (DF) algorithm decodes the received signal at the relay, then re-encodes and retransmits this information, establishing a regenerative system. Outage probabilities, mutual information calculations and transmit diversity bounds for orthogonal amplify and forward (AF) and DF relaying are derived in [18] with the end-to-end performance being considered in [19] where $\mathrm{DF}$ is shown to perform better in terms of the ABER when compared to AF. However, the ABER of regenerative systems depends on the ABER on the individual links. In particular, since SM is shown to outperform other spatial multiplexing techniques on a single link, the application of SM to relaying systems is also shown to provide significant signal-to-noise ratio (SNR) gains when compared to orthogonal DF [20]. Nonetheless, these results are only applicable in a noise-limited relaying system. The deployment of relaying systems around the cell edges, however, may result in interference-limited systems. Therefore, to enable the deployment of SM in a relaying scenario, the ABER performance of SM on a single link must also be assessed in the interference-limited environment.

Most contributions thus far, however, have only addressed SM aspects for point-to-point communication systems, i.e. the single user scenario. Notable exceptions are given in $[21,22]$, where the authors focus their analysis on scenarios employing SSK. The aim of this study is to characterise the behaviour of SM in the multi-user, interference-limited scenario and compare it to the complexity and cost equivalent multi-user MIMO system. We emphasise that SM requires only a single radio frequency (RF) chain at the transmit side since only one is active at any particular instance. Requiring only a single RF chain at the transmitter means that multi-user SM is not comparable in terms of cost to the more complicated spatial-multiplexing multi-user systems analysed in [23-26].

Furthermore, the study in [27] shows that the most energy consuming part of a wireless base station is the power amplifiers and consequently RF chains associated with each transmitter. The study in [28] demonstrates that the power requirements of a base station increase linearly with the number of RF chains added. In addition to higher power consumption, multiple RF chains imply higher manufacturing costs and inter-antenna synchronisation problems. To this extent, SM is an optimal system for utilising the advantages of multiple transmit antennas while still maintaining a single RF chain for Green communications. The aggregate power usage in a system employing SM is significantly lower than a system employing classical MIMO techniques. Furthermore, the lower detection complexity for SM reduces mobile station power usage, enabling a longer battery life for the mobile terminal [9]. Understanding the performance of SM in a multi-user system is necessary to assess its suitability for practical deployment scenarios. In this context, it is of interest if the particular structure of the SM encoding scheme can be exploited to devise novel multi-user detection techniques.

In this study, we first characterise the performance of a single user detector as applied in an interference-limited scenario, i.e. we analyse a ML interference-unaware optimal receiver. We then propose an ML detector which can successfully decode incoming data in the multi-user scenario and is not interference limited, i.e. an interferenceaware detector which can successfully decode data from several nodes. For each detector, we develop an analytical framework to support simulation results and closed form solutions are provided to compute the ABER over identical and independently distributed (i.i.d.) Rayleigh fading channels.

The remainder of this article is organised as follows. In the "System model" section, the system and channel models are introduced. In the "Analytical modelling and receiver design" section, the performance of SM in the multiple access scenario is characterised and the analytical modelling for the multi-user detector is proposed. The "Simulation results and discussion" section provides simulation results to substantiate the accuracy of the developed analytical framework. In the "Summary and conclusions" section, we summarise and conclude this study.

\section{System model}

The basic idea of SM is to map blocks of information bits onto two information carrying units [3]: (i) a symbol, chosen from a complex signal-constellation diagram, and (ii) a unique transmit-antenna, chosen from the set of transmitantennas in an antenna-array, i.e. the spatial-constellation. Jointly, the spatial and signal constellation symbols form a single SM constellation symbol. If, for example, we wish to transmit a total of $4 \mathrm{bits} / \mathrm{s} / \mathrm{Hz}$ using SM with four available transmit antennas; then the first 2 bits would define the spatial-constellation point identifying the active antenna, while the remaining 2 bits would determine the signal-constellation point that will be transmitted.

In the following work, we assume multiple nodes/users, as shown in Figure 1. A total of $N_{u}$ transmit nodes, denoted as $\left\{1, \ldots, \xi, \ldots N_{u}\right\}$, broadcast simultaneously on the same time-frequency slot to a single receiver. Each node broadcasts a signal constellation symbol, $x^{(u)}$, from one of its available antennas.

The received signal at antenna $r$ is given by

$$
y_{r}=\sum_{u=1}^{N_{u}}\left[\sqrt{E_{m} \alpha_{(u)}^{2}} h_{n_{t}^{(u)}, r} x^{(u)}\right]+\eta_{r},
$$




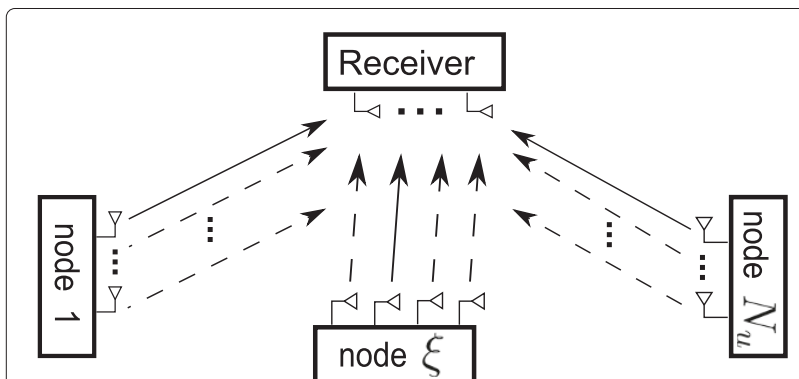

Figure 1 Multi-user SM system setup. Nodes $1, \ldots, \xi, \ldots N_{u}$ broadcast simultaneously to the receiver on the same time-frequency resource block. Each solid line represents the active transmit antenna on each node to every receiving antenna. Each dashed line represents the inactive channel from every transmit antenna at a particular node to every receiving antenna.

where $E_{m}$ is the average transmit energy per symbol, $n_{t}^{(u)}$ is the index of the active transmit antenna from a total of $N_{t}^{(u)}$ available on node $u, r$ is the index of the receive antenna from a total of $N_{r}$ available on the receiving node, $\alpha_{(u)}^{2}$ is the power of the channel attenuation coefficients between all receive antennas and all transmit antennas on the link between node $u$ and the receiver, $h_{n_{t}^{(u)}, r}$ is the fast fading channel coefficient between the active transmit antenna $n_{t}$ on node $u$ and the receiving antenna $r, x^{(u)}$ is the signal constellation symbol transmitted from the set of all possible signal constellation points, $\mathcal{X}^{(u)}$, for node $u$ and $\eta_{r}$, is additive white Gaussian noise (AWGN), defined as a complex normally random variable with zero mean and variance $N_{o}, \mathcal{C N}\left(0, N_{o}\right)$.

Throughout the study $\mathrm{E}_{\mathbf{X}}\left[|x|^{2}\right]=1$, meaning the average power in the signal constellation $\mathcal{X}^{(u)}$ is normalised to one for all $u$. To avoid repetitive definitions of symbols, we note that symbols denoted with $\hat{\imath}$ are simply an element of the same set as the symbol without $\hat{\imath}$, i.e. $\hat{x}$ comes from the same set as $x$. Furthermore, all bold letters are vectors. If we set the signal constellation to be only a single constellation point, where $N_{t}$ is chosen such that $\log _{2}\left(N_{t}\right)$ equals the spectral efficiency, then all presented work can directly be applied to any system employing SSK by simply replacing $x^{(u)}=1$.

\section{Analytical modelling and receiver design}

We analyse the ML detector for use in the multiple access scenario. The detector computes the Euclidean distance between the received vector signal, $y$, and the set of all possible received signals, selecting the closest one.

\section{Interference-unaware detection}

Starting from the system model presented in the "System model" section, the decoded pair $\left(x_{\mathrm{est}}, n_{t}\right)^{(\xi)}$, formed from the estimated symbol $x_{\text {est }}$ emitted from antenna $n_{t}$ on node $\xi$ is given by

$$
\begin{gathered}
\left(x_{\text {est }}, n_{t}\right)^{(\xi)}=\operatorname{argmin}\left\{\left\|\mathbf{y}-\sqrt{E_{m} \alpha_{(\xi)}^{2}} x^{(\xi)} \mathbf{h}_{n_{t}^{(\xi)}}\right\|_{\mathrm{F}}^{2}\right\}, \\
x_{\text {est }}^{(\xi)} \in \mathcal{X}^{(\xi)}, \quad n_{t}^{(\xi)} \in\left\{1 \ldots N_{t}^{(\xi)}\right\}, \quad \mathbf{h}_{n_{t}^{(\xi)}}=\left[h_{n_{t}^{(\xi)}, 1} \ldots h_{n_{t}^{(\xi)}, N_{r}}\right],
\end{gathered}
$$

where $N_{t}^{(\xi)}$ is the number of available transmit antennas on node $\xi,\|\cdot\|_{\mathrm{F}}$ is the Frobenius norm and $\mathcal{X}^{(\xi)}$ has a total of $M^{(\xi)}$ constellation points. We note that $u$ represents the index of a general node in the system, and $\xi$ is the index of the desired node whose data stream is being decoded.

We can use union bound techniques to describe the behaviour of the interference-unaware SM detector in the high SNR regions. From here, we proceed to characterise the behaviour of the interference-unaware detector by defining

$$
\begin{aligned}
& \mathbf{A}=\sqrt{E_{m} \alpha_{(\xi)}^{2}} \mathbf{h}_{n_{t}^{(\xi)}} x^{(\xi)}, \\
& \mathbf{B}=\sum_{u \neq \xi=1}^{N_{u}} \sqrt{E_{m} \alpha_{(u)}^{2}} \mathbf{h}_{n_{t}^{(u)}} x^{(u)},
\end{aligned}
$$

such that

$$
\begin{aligned}
& A_{r}=\sqrt{E_{m} \alpha_{(\xi)}^{2}} h_{n_{t}^{(\xi)}, r} x^{(\xi)}, \text { and } \\
& B_{r}=\sum_{u \neq \xi=1}^{N_{u}} \sqrt{E_{m} \alpha_{(u)}^{2}} h_{n_{t}^{(u)}, r} x^{(u)},
\end{aligned}
$$

define the symbols at the receive antenna $r$. With this, we can pose $\mathbf{y}=\mathbf{A}+\mathbf{B}+\boldsymbol{\eta}$.

The pairwise error probability (PEP) can now be derived as

$$
\begin{aligned}
& \operatorname{Pr}\{\mathbf{A} \neq \hat{\mathbf{A}} \mid \mathbf{B}\}=\operatorname{Pr}\left\{\|\mathbf{y}-\mathbf{A} \mid\|_{\mathrm{F}}^{2}>\|\mathbf{y}-\hat{\mathbf{A}}\|_{\mathrm{F}}^{2}\right\} \\
& =\operatorname{Pr}\left\{\sum_{r=1}^{N_{r}}\left[\left|y_{r}-A_{r}\right|^{2}\right]>\sum_{r=1}^{N_{r}}\left[\left|y_{r}-\hat{A}_{r}\right|^{2}\right]\right\} \\
& =\operatorname{Pr}\left\{\sum_{r=1}^{N_{r}}\left[2 \Re\left\{\left(\hat{A}_{r}-A_{r}\right)\left(B_{r}+\eta_{r}\right)^{*}\right\}\right]>\sum_{r=1}^{N_{r}}\left[\left|A_{r}-\hat{A}_{r}\right|^{2}\right]\right\} \\
& \Rightarrow \eta_{r}^{\text {new }} \sim \mathcal{N}\left(\sum_{r=1}^{N_{r}}\left[2 \Re\left\{\left(\hat{A}_{r}-A_{r}\right) B_{r}^{*}\right\}\right], \sum_{r=1}^{N_{r}}\left[2 N_{o}\left|\hat{A}_{r}-A_{r}\right|^{2}\right]\right),
\end{aligned}
$$

where $(\cdot)^{*}$ represents the complex conjugate, $\eta_{r}^{\text {new }}$ is the distribution of the PEP defined by $\mathcal{N}\left(\mu, \sigma^{2}\right)$ which represents the normal distribution with mean $\mu$ and variance $\sigma^{2}$. Knowing that $\eta_{r}^{\text {new }}$ is the only remaining random variable with a known distribution, enables us to define the 
PEP in (4) using the $Q$-function. Considering $\eta_{r}^{\text {new }}$ and (4), we can see that the PEP can be defined as

$$
\operatorname{Pr}\{\mathbf{A} \neq \hat{\mathbf{A}} \mid \mathbf{B}\}=Q\left(\frac{\sum_{r=1}^{N_{r}}\left[\left|A_{r}-\hat{A}_{r}\right|^{2}-2 \Re\left\{\left(\hat{A}_{r}-A_{r}\right) B_{r}^{*}\right\}\right]}{\sqrt{\sum_{r=1}^{N_{r}}\left[2 N_{o}\left|A_{r}-\hat{A}_{r}\right|^{2}\right]}}\right),
$$

where $Q(\omega)=\frac{1}{\sqrt{2 \pi}} \int_{\omega}^{\infty} \exp \left(-\frac{t^{2}}{2}\right) \mathrm{dt}$ defines the $Q$ function. It should be noted that this PEP is valid for all channel fading statistics. We can further simplify it if we assume that the fast fading channel statistics of each element of $\mathbf{B}$ are Rayleigh distributed, i.e. $h_{n_{t}^{(u)}, r} \sim \mathcal{C N}(0,1)$ and $\mathbf{h}_{n_{t}^{(u)}}$ has $N_{r}$ number of elements. To this end, we define

$$
\begin{aligned}
& \Omega_{P}=\|\mathbf{A}-\hat{\mathbf{A}}\|_{\mathbf{F}^{\prime}}^{2}, \quad \Omega_{I}=2 \Re\left\{(\hat{\mathbf{A}}-\mathbf{A}) \mathbf{B}^{*}\right\}, \\
& \Omega_{N}=\sqrt{2 N_{o} \Omega_{P}},
\end{aligned}
$$

where we can see that

$$
\Omega_{I} \sim \mathcal{N}\left(0,2 E_{m}\left|x_{\mathbf{B}}\right|^{2} \Omega_{P}\right),
$$

such that $\left|x_{\mathbf{B}}\right|^{2}=\sum_{u \neq \xi=1}^{N_{u}} \alpha_{(u)}^{2}\left|x^{(u)}\right|^{2}$ and define $\sigma_{I}^{2}=2 E_{m}\left|x_{\mathbf{B}}\right|^{2} \Omega_{P}$. We can remove the channel effects of $\mathbf{B}$ from the expression by taking the expectation of (5) across the fading channel $\mathbf{h}_{\mathbf{B}}$, such that

$$
\begin{aligned}
\operatorname{Pr}\{\mathbf{A} \neq \hat{\mathbf{A}}\} & =\mathrm{E}_{\mathbf{h}_{\mathbf{B}}}\left[\operatorname{Pr}\left\{\mathbf{A} \neq \hat{\mathbf{A}} \mid \mathbf{h}_{\mathbf{B}}\right\}\right], \\
\operatorname{Pr}\{\mathbf{A} \neq \hat{\mathbf{A}}\} & =\mathrm{E}_{\mathbf{h}_{\mathbf{B}}}\left[Q\left(\frac{\Omega_{P}-\Omega_{I}}{\Omega_{N}}\right)\right], \\
& =\mathrm{E}_{\mathbf{h}_{\mathbf{B}}}\left[Q\left(\frac{\Omega_{P}}{\Omega_{N}}-\frac{\sigma_{I}}{\Omega_{N}} \hat{\Omega}_{I}\right)\right],
\end{aligned}
$$

where $\hat{\Omega}_{I} \sim \mathcal{N}(0,1)$ and $E_{\mathbf{h}}[\cdot]$ represents the expectation of the system with respect to the fast fading channel $\mathbf{h}$. From here, we can apply ([29], eq. 3.66) which results in

$$
\operatorname{Pr}\{\mathbf{A} \neq \hat{\mathbf{A}}\}=Q\left(\frac{\Omega_{P}}{\sqrt{\Omega_{N}^{2}+\sigma_{I}^{2}}}\right) .
$$

After some analytical manipulations we obtain (10)

$$
\operatorname{Pr}\{\mathbf{A} \neq \hat{\mathbf{A}}\}=Q\left(\sqrt{\gamma_{\mathbf{I}}\left\|\mathbf{h}_{n_{t}^{(\xi)}} x^{(\xi)}-\mathbf{h}_{\hat{n}_{t}^{(\xi)}} \hat{x}^{(\xi)}\right\|_{\mathrm{F}}^{2}}\right),
$$

where

$$
\gamma_{\mathbf{I}}=\frac{1}{2} \frac{E_{m} \alpha_{(\xi)}^{2}}{N_{o}+E_{m} \sum_{u \neq \xi=1}^{N_{u}} \alpha_{(u)}^{2}\left|x^{(u)}\right|^{2}},
$$

represents half of the signal-to-interference-plus-noise ratio (SINR) between node $\xi$ and the receiver. Throughout the study, we average only across the fast fading channel statistics. As (11) shows, $\gamma_{I}$ is still dependent on the magnitude of the modulated signal symbols of the interfering nodes, $\left|x^{(u)}\right|$. This means that all expressions using $\gamma_{\text {I }}$ maintain their conditioning on the modulated signal symbols of the interfering nodes.

Given this formulation, we can now define the ABER of the single-user detector using the union bounding techniques in the presence of interference as

$$
\begin{aligned}
& \operatorname{ABER}_{\xi}^{(\text {inter) }} \leq \sum_{\substack{x^{(\xi)}, n_{t}^{(\xi)} \\
\hat{x}^{(\xi)}, \hat{n}_{t}^{(\xi)}}}^{\sum \sum \cdots \sum} \frac{d_{\xi}(b, \hat{b})}{\left.\operatorname{lN}_{u}-1\right) \text { summations }} \\
& \times \frac{\mathrm{E}_{\mathbf{h}_{\mathbf{A}}}[\operatorname{Pr}\{\mathbf{A} \neq \hat{\mathbf{A}}\}]}{M^{(\xi)} N_{t}^{(\xi)}}\left[\prod_{u \neq \xi=1}^{N_{u}} \frac{1}{\left.M^{(\xi)} N_{t}^{(\xi)}\right)}\right],
\end{aligned}
$$

where the $u$ th summation from the $\left(N_{u}-1\right)$ summations above is defined for all $x^{(u)} \in \mathcal{X}^{(u)}$ and $u \neq$ $\xi$ with $M^{(\xi)}$ being the cardinality of $\mathcal{X}^{(\xi)}$. The symbol $\sum_{x^{(\xi)}, n_{t}^{(\xi)}}^{M^{(\xi)} N_{t}^{(\xi)}}$ is defined as a fourfold summation, two for

$$
\hat{x}^{(\xi)}, \hat{n}_{t}^{(\xi)}
$$

all $x^{(\xi)}, \hat{x}^{(\xi)} \in \mathcal{X}^{(\xi)}$ and two for the indices $n_{t}^{(\xi)}, \hat{n}_{t}^{(\xi)} \in$ $\left[1, \ldots, N_{t}^{(\xi)}\right]$. In addition, $d_{\xi}(b, \hat{b})=d_{\xi}\left(n_{t}, \hat{n}_{t}\right)+d_{\xi}(x, \hat{x})$, where $d_{\xi}(\cdot, \hat{\imath})$ denotes the Hamming distance between the binary representations any two symbols coming from the same set for node $\xi$.

As with the interfering nodes, we assume that the desired node's fast fading follows a Rayleigh distribution. To obtain the average PEP, we define $z_{r}=h_{n_{t}^{(\xi)}, r} x_{(\xi)}-h_{\hat{n}_{t}^{(\xi)}, r} \hat{x}_{(\xi)}$, with a variance of

$$
\sigma_{z}^{2}= \begin{cases}\left(\left|x^{(\xi)}\right|^{2}+\left|\hat{x}^{(\xi)}\right|^{2}\right) & n_{t}^{(\xi)} \neq \hat{n}_{t}^{(\xi)}, \\ \left(\left|x^{(\xi)}-\hat{x}^{(\xi)}\right|^{2}\right) & n_{t}^{(\xi)}=\hat{n}_{t}^{(\xi)}, \\ 0 & n_{t}^{(\xi)}=\hat{n}_{t}^{(\xi)} \text { and } x^{(\xi)}=\hat{x}^{(\xi)},\end{cases}
$$

where $\sigma_{z}^{2}$ is the variance of SM using a variable amplitude modulation scheme. In particular, it is the variance per receive antenna of the argument inside the $Q$-function in (10). We define the random variable

$$
\kappa=\frac{\|\left.\mathbf{z}\right|_{\mathrm{F}} ^{2}}{\gamma_{\mathbf{I}} \sigma_{z}^{2} / 2}=\sum_{r=1}^{N_{r}}\left|\frac{z_{r}}{\gamma_{\mathbf{I}} \sigma_{z} / \sqrt{2}}\right|^{2},
$$

which has a central Chi-squared distribution with $2 N_{r}$ degrees of freedom given as

$$
\tilde{\rho}_{K}(\kappa)=\frac{1}{2^{N_{r}}\left(N_{r}-1\right) !} \kappa^{N_{r}-1} e^{-\kappa / 2}
$$


where $(\cdot)$ ! represents the factorial function. We can now pose

$$
\begin{aligned}
\mathrm{E}_{\mathbf{h}_{\mathbf{A}}}[\operatorname{Pr}\{\mathbf{A} \neq \hat{\mathbf{A}}\}]= & \frac{(1 / 2)^{N_{r}}}{\left(N_{r}-1\right) !} \\
& \times \int_{0}^{\infty} e^{-\kappa / 2} \kappa^{N_{r}-1} Q\left(\sqrt{\frac{\gamma_{\mathbf{I}} \sigma_{z}^{2}}{2} \kappa}\right) \mathrm{d} \kappa .
\end{aligned}
$$

By direct inspection, we can now apply the solution to ([30], eq. 62) and obtain

$$
\mathrm{E}_{\mathbf{h}_{\mathbf{A}}}[\operatorname{Pr}\{\mathbf{A} \neq \hat{\mathbf{A}}\}]=f(\beta)^{N_{r}} \sum_{r=0}^{N_{r}-1}\left(\begin{array}{c}
N_{r}-1+r \\
r
\end{array}\right)(1-f(\beta))^{r},
$$

where

$$
f(\beta)=\frac{1}{2}\left(1-\sqrt{\frac{\beta}{1+\beta}}\right),
$$

and

$$
\beta=\frac{\gamma_{1} \sigma_{z}^{2}}{2} .
$$

We note that (12) presents an analytical bound for a system employing quadrature-amplitude-modulation (QAM). If we choose to use constant-amplitude modulation such as phase-shift-keying (PSK), then for a fixed channel realisation, we define $a=h_{n_{t}^{(\xi)}, r}, b=x_{(\xi)}$, and pose

$$
z_{r}^{\mathrm{PSK}}=|a| e^{j \arg (a)}|b| e^{j \arg (b)}-|\hat{a}| e^{j \arg (\hat{a})}|\hat{b}| e^{j \arg (\hat{b})},
$$

where $\arg (\cdot)$ represents the phase of a complex symbol. We realise that the amplitude of all constellation points is unity and thus

$$
z_{r}^{\mathrm{PSK}}=|a| e^{j(\arg (a)+\arg (b))}-|\hat{a}| e^{j(\arg (\hat{a})+\arg (\hat{b}))} .
$$

From the definition of $z_{r}^{\mathrm{PSK}}$, it is clear that its variance is defined as

$$
\sigma_{z^{\mathrm{PKK}}}^{2}=\left\{\begin{array}{l}
2 \text { if } n_{t}^{(\xi)} \neq \hat{n}_{t}^{(\xi)} \text { or } \arg \left(x_{(\xi)}\right) \neq \arg \left(\hat{x}_{(\xi)}\right), \\
0 \text { if } n_{t}^{(\xi)}=\hat{n}_{t}^{(\xi)} \text { and } \arg \left(x_{(\xi)}\right)=\arg \left(\hat{x}_{(\xi)}\right) .
\end{array}\right.
$$

In this case, (12) reduces to

$$
\begin{aligned}
\operatorname{ABER}_{\xi}^{(\text {PSK-inter) }} \leq & \sum_{n_{t}=1}^{N_{t}^{(\xi)}} \sum_{\hat{n}_{t}=1}^{N_{t}^{(\xi)}} \frac{d_{\xi}(b, \hat{b})}{\log _{2}\left(M^{(\xi)} N_{t}^{(\xi)}\right)} \\
& \times \frac{E_{\mathbf{h}_{\mathbf{A}}}^{\text {PSK }}[\operatorname{Pr}\{\mathbf{A} \neq \hat{\mathbf{A}}\}]}{M^{(\xi)} N_{t}^{(\xi)}},
\end{aligned}
$$

where

$$
\begin{aligned}
\mathrm{E}_{\mathbf{h}_{\mathbf{A}}}^{\mathrm{PSK}}[\operatorname{Pr}\{\mathbf{A} \neq \hat{\mathbf{A}}\}]= & f\left(\beta^{\mathrm{PSK}}\right)^{N_{r}} \sum_{r=0}^{N_{r}-1}\left(\begin{array}{c}
N_{r}-1+r \\
r
\end{array}\right) \\
& \times\left(1-f\left(\beta^{\mathrm{PSK}}\right)\right)^{r},
\end{aligned}
$$

such that

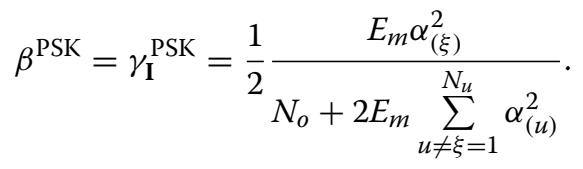

The average power carried by any signal-symbol constellation in (12) is 1 . Nonetheless, a variable amplitude modulation scheme means that the instantaneous SINR changes. The instantaneous SINR must strictly be defined to study the asymptotic behaviour of the system. This is necessary because the instantaneous SINR is an argument of the PEP which is defined using the $Q$-function. To obtain the ABER, the PEP must be averaged across all channel realisations and all signal-symbol constellations. A closed-form expression for the asymptotic behaviour of (12) and (13) is therefore difficult to obtain. However, we note that (22) and (23) are special cases of (12) and (13) which enable a simpler theoretical analysis of the asymptotic behaviour of the system as the SINR grows to infinity. Simulation results in the "Simulation results and discussion" section show that the asymptotic bounds derived for SM using a constant amplitude modulation scheme are also valid for SM using 4-QAM. In addition, it is shown that for the point-to-point single user scenario, SM using PSK may have a better ABER performance than SM using QAM depending on the number of available transmit antennas [31]. We now proceed with the asymptotic analysis of this system.

\section{Asymptotic analysis of an interference-unaware detector} In this section, we investigate some asymptotic cases to highlight trends in SM at high SNR. Simulations in the "Simulation results and discussion" section show that the presented results are asymptotically tight in the high SNR region. We first define $\mathrm{SNR}_{\xi}=E_{m} \alpha_{(\xi)}^{2} /\left(2 N_{o}\right)$ and $\operatorname{SIR}_{\xi}=\frac{\alpha_{(\xi)}^{2}}{\sum_{u \neq \xi=1}^{N_{u}} \alpha_{(u)}^{2}\left|x^{(u)}\right|^{2}}$. With these definitions, we look at three systems, the asymptotic performance of SM and SIMO in the noise-limited scenario and the asymptotic performance of SM in the interference-limited scenario.

\section{$S N R_{\xi} \gg 1$ and $\operatorname{SINR} \approx S N R$ (noise-limited scenario)}

This is the classic single user scenario where multiple access interference can be neglected, and high-SNR analysis for the probe link can be considered. We look at (12), 
where in the limit $\gamma_{\mathbf{I}} \approx \gamma=E_{m} \alpha_{(\xi)}^{2} /\left(2 N_{o}\right)$. Since interference can be neglected,

$$
\begin{aligned}
\lim _{\gamma \rightarrow \infty} \operatorname{ABER}_{\xi}^{(\text {inter })}= & \lim _{\gamma \rightarrow \infty} \sum_{\substack{x^{(\xi)}, n_{t}^{(\xi)}, \hat{x}^{(\xi)}, \hat{n}_{t}^{(\xi)}}}^{\log _{2}^{(\xi)}\left(M^{(\xi)} N_{t}^{(\xi)}\right)} \\
& \times \frac{\mathrm{E}_{\mathbf{h}_{\mathbf{A}}}[\operatorname{Pr}\{\mathbf{A} \neq \hat{\mathbf{A}}\}]}{M^{(\xi)} N_{t}^{(\xi)}} .
\end{aligned}
$$

To simplify (26), the limit in (17) and (24) can be tackled by considering a Taylor expansion with two terms of (18) and obtain

$$
\begin{aligned}
& f(\beta)=\frac{1}{2}\left(1-\sqrt{\frac{\beta}{1+\beta}}\right) \Rightarrow \text { Taylor expansion } \\
& f(\beta) \approx \frac{1}{2}\left(\frac{1}{2(\beta+1)}\right)=2^{-2}(\beta+1)^{-1} .
\end{aligned}
$$

We realise that the average symbol-error-ratio (ASER) for SM is defined similar to the ABER and can be posed as

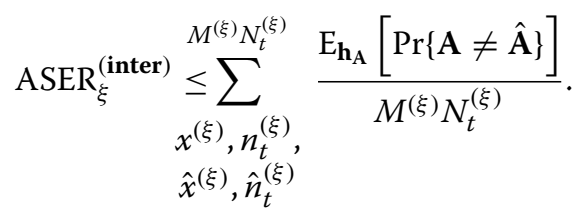

From here, we know that $\mathrm{ABER}_{\xi}^{(\text {inter })} \leq \mathrm{ASER}_{\xi}^{\text {(inter) }} / 2$. In particular, this is a close approximation for ABER of SM, since the fast fading channel coefficients effectively make the SM constellation completely random at the receiver. This negates any benefits from advanced bit-to-symbol mappings such as Gray coding. In effect, the detector will create a long binary sequence which has at most $50 \%$ bit-errors within the erroneous symbol sequence, i.e. the ABER is bounded to be at most $1 / 2$ of the ASER. The tightness of this bound can be seen in the "Simulation results and discussion" section. This step eliminates the dependence on the Hamming distance between the various SM symbols, which permits us to pose

$$
\begin{aligned}
\lim _{\gamma \rightarrow \infty} \frac{\mathrm{ABER}_{\xi}^{(\text {inter })}}{\gamma_{\mathrm{I}}^{-N_{r}}}= & \lim _{\gamma \rightarrow \infty}\left(\begin{array}{c}
2 N_{r}-1 \\
N_{r}
\end{array}\right) 2^{-2 N_{r}} \\
& \times \frac{M^{(\xi)} N_{t}^{(\xi)}}{2} \mathrm{E}_{x, n_{t}, \hat{x}, \hat{n}_{t}}\left[\left(\frac{\sigma_{z}^{2}}{2}\right)^{-N_{r}}\right],
\end{aligned}
$$

where

$$
\mathrm{E}_{x, n_{t}, \hat{x}, \hat{n}_{t}}\left[\left(\frac{\sigma_{z}^{2}}{2}\right)^{-N_{r}}\right]
$$

is the expectation of $\left(\frac{\sigma_{z}^{2}}{2}\right)^{-N_{r}}$ across the various possibilities of $\sigma_{z}^{2}$ for $x, n_{t}, \hat{x}$ and $\hat{n}_{t}$. We are not aware of a closed form solution to the more generic expression for (30) given a variable-amplitude modulation. However, we can upper bound (30) by setting $\sigma_{z}^{2}=\min \left(\sigma_{z}^{2}, 2\right)$. In particular, the general form of $\sigma_{z}^{2}$ is defined by the underlying SM signal-symbol constellation size, $M^{(\xi)}$. To this extent, expressions for $\sigma_{z}^{2}$ are defined using the upper bound for square QAM constellation sizes. A summary of the derivation of (32) is provided in Appendix.

When $M^{(\xi)}=4$, then

$$
\Psi=\mathrm{E}_{n_{t}, x, \hat{n}_{t}, \hat{x}}\left[\left(\frac{\sigma_{z}^{2}}{2}\right)^{-N_{r}}\right] \leq \frac{4 N_{t}^{(\xi)}+\left(2^{-N_{r}}-2\right)}{4 N_{t}^{(\xi)}} .
$$

When $M^{(\xi)}=16$, then

$$
\Psi=\mathrm{E}_{n_{t}, x, \hat{n}_{t}, \hat{x}}\left[\left(\frac{\sigma_{z}^{2}}{2}\right)^{-N_{r}}\right] \leq \frac{\psi_{1}+\psi_{2}}{\left(16 N_{t}^{(\xi)}\right)^{2}},
$$

where

$$
\begin{aligned}
\psi_{1}= & \left(16(0.2)^{-N_{r}}+32(0.6)^{-N_{r}}+208\right)\left(N_{t}^{(\xi)}\right)^{2}, \\
\psi_{2}= & \left(32(0.2)^{-N_{r}}+36(0.4)^{-N_{r}}-32(0.6)^{-N_{r}}\right. \\
& \left.+32(0.8)^{-N_{r}}-84\right) N_{t}^{(\xi)} .
\end{aligned}
$$

With this in mind, the closed form of the limit is defined as

$$
\lim _{\gamma \rightarrow \infty} \frac{\mathrm{ABER}_{\xi}^{(\text {inter })}}{\gamma^{-N_{r}}}=M^{(\xi)} N_{t}^{(\xi)}\left(\begin{array}{c}
2 N_{r}-1 \\
N_{r}
\end{array}\right) 2^{-\left(2 N_{r}+1\right)} \Psi .
$$

At this point, the work can be simplified by considering a constant-amplitude modulation scheme such as PSK where $\sigma_{z^{\text {PSK }}}^{2}$ is either 2 or 0 as shown in (22), then the expression in (30) is unity which simplifies further analysis. In such a scenario, however, the study in [31] shows that SM using PSK cannot always guarantee a better performance than SM using QAM. In particular, the authors of [31] demonstrate that there is a crossing point where the ABER of SM using PSK improves over SM using QAM. Nonetheless, there are two conclusions that we can drawn from this limit: (i) the system error increases with the product of the spatial constellation size, $N_{t}^{(\xi)}$, and symbol constellation size, $M^{(\xi)}$, (ii) the system error decreases exponentially with the addition of each received antenna, i.e. the diversity order is equal to $N_{r}$. In addition, we quantify the coding gains with respect to the 
number of receive antennas as $\left(\begin{array}{c}2 N_{r}-1 \\ N_{r}\end{array}\right) 2^{-\left(2 N_{r}+1\right)}$. This is apparent considering

$$
\left(\begin{array}{c}
2 N_{r}-1 \\
N_{r}
\end{array}\right)<\left(\begin{array}{c}
2 N_{r} \\
N_{r}
\end{array}\right) \ll \sum_{r=0}^{2 N_{r}}\left(\begin{array}{c}
2 N_{r} \\
r
\end{array}\right)=2^{2 N_{r}}<2^{2 N_{r}+1} .
$$

In general, it can be shown that as $N_{r} \rightarrow \infty$, the inverse of (35) tends to zero and is always less than 1 . Since the inverse of (35) is always less than 1, the addition of an extra receive antenna implies a smaller ratio, which means a lower ABER and hence coding gains for the system.

\section{SIMO system utilising QAM (noise-limited scenario)}

To quantify the SNR difference between SM and SIMO using QAM, we need to look at the ABER performance of a SIMO system using QAM in the asymptote. We use ([32], eq. 9.23) which provides a closed form solution for the ASER of a SIMO system using QAM with i.i.d. inputs, i.e. no correlation between the receive antennas. To begin the asymptotic analysis, ([32], eq. 9.23) is tightly upper bounded by

$$
\begin{aligned}
\operatorname{ASER}_{\mathrm{QAM}} \leq & 4\left(1-\frac{1}{\sqrt{\widetilde{M}}}\right) f\left(\gamma_{\mathrm{QAM}}\right)^{N_{r}} \\
& \times \sum_{r=0}^{N_{r}-1}\left(\begin{array}{c}
N_{r}-1+r \\
r
\end{array}\right)\left(1-f\left(\gamma_{\mathrm{QAM}}\right)\right)^{r},
\end{aligned}
$$

where $\gamma_{\mathrm{QAM}}=\frac{3}{2(\tilde{M}-1)} \gamma$. The interested reader is invited to look at the work in [32] for more details in obtaining (36). We can now pose ASER $\mathrm{QAM} / \log _{2}(\widetilde{M}) \approx \mathrm{ABER}_{\mathrm{QAM}}$ provided Gray mapping is used ([32], eq. 8.7), where $\widetilde{M}=M^{(\xi)} N_{t}^{(\xi)}$.

Using (27) and evaluating (36) in the limit as $\gamma$ tends to infinity, it reduces to

$$
\begin{aligned}
\lim _{\gamma \rightarrow \infty} \frac{\mathrm{ABER}_{\mathrm{QAM}}}{\gamma^{-\left(N_{r}\right)} \leq} & \frac{4}{\log _{2}(\widetilde{M})}\left(1-\frac{1}{\sqrt{\widetilde{M}}}\right) \\
& \times\left(\frac{3}{2(\widetilde{M}-1)}\right)^{-N_{r}}\left(\begin{array}{c}
2 N_{r}-1 \\
N_{r}
\end{array}\right) 2^{-\left(2 N_{r}\right)} .
\end{aligned}
$$

If we look at the ratio of (34) with (31) to (37) then, after some analytical manipulations shown in the "Reaching (38) from (34) with (31) and (37)" section in the Appendix, we can pose

$$
\lim _{\gamma \rightarrow \infty} \frac{\mathrm{ABER}_{\xi}^{(\text {inter) }} / \gamma^{-N_{r}}}{\mathrm{ABER}_{\mathrm{QAM}} / \gamma^{-N_{r}}}=\frac{\left(2 N_{t}^{(\xi)}+2^{-N_{r}-1}-1\right)}{\left(\frac{2\left(4 N_{t}^{(\xi)}-1\right)}{3}\right)^{N_{r}} \frac{4}{\log _{2}\left(4 N_{t}^{(\xi)}\right)}\left(1-\frac{1}{\sqrt{4 N_{t}^{(\xi)}}}\right)},
$$

for $M^{(\xi)}=4$. If we equate the right-hand side of (38) to 1 , then the expression cannot be solved in closed form. However, since $N_{r}$ and $N_{t}^{(\xi)}$ are natural numbers we can readily evaluate (38). When evaluating (38), if the result is greater than 1, then SIMO transmission using only QAM is better than using SM. If the result is less than 1, then transmission using SM performs better than transmission using only QAM. The results for $N_{t}^{(\xi)}=2^{q}$ where $q \in\{1, \ldots, 6\}$ and $N_{r} \in\{1,2,3\}$ are presented in Table 1 , which shows that $\mathrm{SM}$ is always better if $N_{r} \geq 2$. In addition, the ratio of the two, as given in Table 1, exactly quantifies the minimum coding gain of SM relative to QAM for the same spectral efficiency of $\log _{2}\left(M^{(\xi)} N_{t}^{(\xi)}\right)$ in a noise-limited scenario, given $M^{(\xi)}=4$.

Proceeding in a similar manner as for (38), we pose the general ratio of the relative coding gains achieved by SM, using a variable-amplitude modulation, over a SIMO system using QAM as

$$
\lim _{\gamma \rightarrow \infty} \frac{\mathrm{ABER}_{\xi}^{(\text {inter })} / \gamma^{-\left(N_{r}\right)}}{\mathrm{ABER} \mathrm{QAM}_{\mathrm{QAM}} / \gamma^{-\left(N_{r}\right)}}=\frac{\tilde{M} \Psi}{\left(\frac{2(\widetilde{M}-1)}{3}\right)^{N_{r}} \frac{2}{\log _{2}(M)}\left(1-\frac{1}{\sqrt{M}}\right)},
$$

where $\Psi$ must be defined for the desired SM signal constellation size, $M^{(\xi)}$. The exact ratios as given in Table 1 will vary depending on $M^{(\xi)}$, but the trend (SM outperforming SIMO) will remain, as can be seen in Table 2 for $M^{(\xi)}=16$, i.e. the values in the last two rows of the tables are smaller than one.

Similarly, using a constant-amplitude modulation scheme such as PSK, we realise that the only difference is that $\Psi$ will be unity in (39). Implementing this change means removing the dependence on the signal constellation size for SM and resulting in a single table of values for (39), as given in Table 3.

From Tables 1, 2, and 3, we can conclude that a singleinput-single-output system using QAM performs better than SM using QAM or PSK, i.e. the values in the first row of each table are greater than 1 . These results are applicable only to SM which means that at least 1 bit must be sent via the signal-symbol.

\section{$S I R_{\xi} \gg 1$ and $S I N R \approx S I R_{\xi}$ (interference-limited scenario)}

In this case, we neglect the AWGN in the channel and realise that the $\operatorname{SIR}_{\xi}$ is the dominant term dictating the ABER performance of the system. Looking at the expression for the SIR, we realise that $\gamma_{I}$ is a function of the signal-symbol amplitude for all users and their respective channel attenuations. Thus, we cannot simply extract $\gamma_{\mathbf{I}}$ from (34). In particular, in the expression for $\gamma_{\mathbf{I}}$ in (11), $\left|x_{(u)}\right|$ may be greater than 1, which would increase the interference from the remaining users. Due to the complexity of the expressions, further asymptotic study of the 
Table 1 Relative coding gains of SM using 4-QAM compared to SIMO using $\tilde{M}$-QAM

\begin{tabular}{lllllll}
\hline $\boldsymbol{N}_{\boldsymbol{r}}$ & $\boldsymbol{N}_{\boldsymbol{t}}^{(\xi)}=\mathbf{2}^{\mathbf{1}}$ & $\boldsymbol{N}_{\boldsymbol{t}}^{(\xi)}=\mathbf{2}^{\mathbf{2}}$ & $\boldsymbol{N}_{\boldsymbol{t}}^{(\xi)}=\mathbf{2}^{\mathbf{3}}$ & $\boldsymbol{N}_{\boldsymbol{t}}^{(\xi)}=\mathbf{2}^{\mathbf{4}}$ & $\boldsymbol{N}_{\boldsymbol{t}}^{(\xi)}=\mathbf{2}^{\mathbf{5}}$ & $\boldsymbol{N}_{\boldsymbol{t}}^{(\xi)}=\mathbf{2}^{\mathbf{6}}$ \\
\hline 1 & 1.6 & 1.9 & 2.3 & 2.6 & 2.9 & 3.2 \\
2 & 0.67 & 0.38 & 0.22 & 0.12 & 0.068 & 0.038 \\
3 & 0.28 & 0.075 & 0.021 & 0.0057 & 0.0016 & $4.4\left(10^{-4}\right)$ \\
\hline
\end{tabular}

interference-limited scenario for SM will be constrained to using a constant-amplitude modulation scheme such as PSK. SM using PSK is shown to perform better than SM using QAM in certain cases [31]. For SM using PSK,

$$
\gamma_{\mathbf{I}}^{\mathrm{PSK}} \approx \frac{\alpha_{(\xi)}^{2}}{2 \sum_{\substack{u \neq \xi=1 \\ N_{u}}}^{2}},
$$

in the interference-limited scenario. We see that the limit of the ABER tends to (34) with a slight, but very important distinction: the system reaches an error floor. This is to be expected when the receiver is interference-unaware. If $\gamma_{\mathbf{I}}$ or $\gamma_{\mathbf{I}}^{\text {PSK }}$ are not large enough, i.e. the channel attenuations of the various nodes are similar, then this study presents an upper bound for the ABER of the system since (34) still defines the behaviour of the system in the limit. There are three consequences that should be considered similar to the noise-limited scenario analysed above: (i) the system error performance improves when more receive antennas are added at the receiver, (ii) the system error performance worsens as more SM constellation points are added, as either $N_{t}^{(\xi)}$ or $M^{(\xi)}$ is increased and (iii) the detector will fail to decode any data emitted from a node whose desired signal is weaker than the interfering signal. Although analytical work for SM using QAM becomes intractable, numerical results demonstrate that SM using a variable-amplitude modulation performs in a similar fashion to SM using PSK and leads to the same conclusions. In the remainder of this study, we show that the near-far problem is completely mitigated by applying a jointly optimal ML detector for SM in an interferencelimited scenario. In other words, all incoming streams will be decoded and, in particular, the error performance of the system will tend to zero as AWGN approaches zero, despite any interference.

\section{Interference-aware detection}

Starting from the system model presented in the "System model" section, the decoded pair $\left(x_{\mathrm{est}}, n_{t}\right)^{(\xi)}$, formed from the estimated symbol $x_{\text {est }}$ emitted from antenna $n_{t}$ on node $\xi$ is given by

$$
\begin{gathered}
\left\{\begin{array}{c}
\left(x, n_{t}\right)^{(1)}, \\
\vdots \\
\left(x, n_{t}\right)^{(\xi)}, \\
\vdots \\
\left(x, n_{t}\right)^{\left(N_{u}\right)},
\end{array}\right\}=\operatorname{argmin}\left\{\left\|\mathbf{y}-\sum_{u=1}^{N_{u}} x^{(u)} \mathbf{h}_{n_{t}^{(u)}}\right\|_{\mathrm{F}}\right\} \\
x^{(u)} \in \mathcal{X}^{(u)} \quad \text { and } \quad n_{t}^{(u)} \in\left\{1, \ldots, N_{t}^{(u)}\right\} .
\end{gathered}
$$

Similar to the work in the "Interference-unaware detection" section, the union bound technique is used to describe the behaviour of the interference-aware SM detector in the high SNR regions. The main difference between the two detectors comes from the computation of the PEP between the possible received symbols. The union bound for the interference-aware SM detector, which estimates the ABER for node $\xi$, can be expressed as

$$
\begin{aligned}
\operatorname{ABER}_{\xi} & \leq \sum_{x^{(1)}, n_{t}^{(1)}, x^{\left(N_{u}\right)}, n_{t}^{\left(N_{u}\right)},}^{M_{t}^{(1)}} \frac{d \xi(b, \hat{b})}{\log _{2}\left(M^{(\xi)} N_{t}^{(\xi)}\right)} \\
\hat{x}^{(1)}, \hat{n}_{t}^{(1)} \hat{x}^{\left(N_{u}\right)}, \hat{n}_{t}^{\left(N_{u}\right)} & \sum_{t}^{\left(N_{u}\right)} \\
& \times \frac{\mathrm{E}_{\mathbf{H}}\left[\operatorname{PEP}\left(x^{(\Omega)}, n_{t}^{(\Omega)}, \hat{x}^{(\Omega)}, \hat{n}_{t}^{(\Omega)}\right)\right]}{\prod_{u=1}^{N_{u}} M^{(u)} N_{t}^{(u)}} .
\end{aligned}
$$

The pairs, $\left(x^{(\Omega)}, n_{t}^{(\Omega)}\right)$ and $\left(\hat{x}^{(\Omega)}, \hat{n}_{t}^{(\Omega)}\right)$, come from the set of all possible symbol-antenna pairings for all nodes, i.e. they independently take values from the set of all possible spatial and signal constellation points, $\Omega$. We define $\operatorname{PEP}\left(x^{(\Omega)}, n_{t}^{(\Omega)}, \hat{x}^{(\Omega)}, \hat{n}_{t}^{(\Omega)}\right)$ to be the PEP between the symbol $x^{(\Omega)}$ emitted from antenna $n_{t}^{(\Omega)}$ being detected as symbol $\hat{x}^{(\Omega)}$ emitted by antenna $\hat{n}_{t}^{(\Omega)}$.

Table 2 Relative coding gains of SM using 16-QAM compared to SIMO using $\tilde{M}$-QAM

\begin{tabular}{lllllll}
\hline $\boldsymbol{N}_{\boldsymbol{r}}$ & $\boldsymbol{N}_{\boldsymbol{t}}^{(\boldsymbol{\xi})}=\mathbf{2}^{\mathbf{1}}$ & $\boldsymbol{N}_{\boldsymbol{t}}^{(\boldsymbol{\xi})}=\mathbf{2}^{\mathbf{2}}$ & $\boldsymbol{N}_{\boldsymbol{t}}^{(\boldsymbol{\xi})}=\mathbf{2}^{\mathbf{3}}$ & $\boldsymbol{N}_{\boldsymbol{t}}^{(\boldsymbol{\xi})}=\mathbf{2}^{\mathbf{4}}$ & $\boldsymbol{N}_{\boldsymbol{t}}^{(\boldsymbol{\xi})}=\mathbf{2}^{\mathbf{5}}$ & $\boldsymbol{N}_{\boldsymbol{t}}^{(\boldsymbol{\xi})}=\mathbf{2}^{\mathbf{6}}$ \\
\hline 1 & 3.8359 & 3.8724 & 4.0859 & 4.4031 & 4.7832 & 5.2024 \\
2 & 0.2551 & 0.1121 & 0.0542 & 0.0278 & 0.0147 & 0.0079 \\
3 & 0.0245 & 0.0050 & 0.0011 & 0.0003 & 0.0001 & $2.0\left(10^{-5}\right)$ \\
\hline
\end{tabular}


Table 3 Relative coding gains of SM using PSK compared to SIMO using $\tilde{M}$-QAM

\begin{tabular}{lllllll}
\hline $\boldsymbol{N}_{\boldsymbol{r}}$ & $\tilde{\mathbf{M}}=\mathbf{2}^{\mathbf{2}}$ & $\tilde{\mathbf{M}}=\mathbf{2}^{\mathbf{3}}$ & $\tilde{\mathbf{M}}=\mathbf{2}^{\mathbf{4}}$ & $\tilde{\mathbf{M}}=\mathbf{2}^{\mathbf{5}}$ & $\tilde{\mathbf{M}}=\mathbf{2}^{\mathbf{6}}$ & $\tilde{\mathbf{M}}=\mathbf{2}^{\mathbf{7}}$ \\
\hline 1 & 2 & 1.9889 & 2.1333 & 2.3511 & 2.6122 & 2.9022 \\
2 & 0.5000 & 0.2131 & 0.1067 & 0.0569 & 0.0311 & 0.0171 \\
3 & 0.1250 & 0.0228 & 0.0053 & 0.0014 & $3.7\left(10^{-4}\right)$ & $1.0\left(10^{-4}\right)$ \\
\hline
\end{tabular}

Similar to the analytical derivation of (4) in the "Inter ference-unaware detection" section, the ABER for node $\xi$ is shown in (42), where the PEP is given as

$$
\begin{aligned}
& \operatorname{PEP}\left(x^{(\Omega)}, n_{t}^{(\Omega)}, \hat{x}^{(\Omega)}, \hat{n}_{t}^{(\Omega)}\right) \\
& =Q\left(\sqrt{\frac{E_{m}}{2 N_{o}}\left\|\sum_{u=1}^{N_{u}} \alpha_{(u)}\left(\mathbf{h}_{n_{t}^{(u)}} x^{(u)}-\mathbf{h}_{\hat{n}_{t}^{(u)}} \hat{x}^{(u)}\right)\right\|_{\mathrm{F}}^{2}}\right) .
\end{aligned}
$$

A more detailed derivation of (43) is given in the "Derivation of (43)" section in the Appendix. We note that thus far no assumptions have been made as to the channel distribution. Considering Rayleigh fading channels for all links in the system, we can derive the closed form solution for $\mathrm{E}_{\mathbf{H}}[\mathrm{PEP}(\cdot)]$ in (42) in the same manner as shown in the "Interference-unaware detection" section with (16) and (17) such that

$$
\beta=\frac{E_{m}}{4 N_{o}} \sum_{u=1}^{N_{u}} \alpha_{(u)}^{2} \vartheta_{(u)}
$$

and

$$
\vartheta_{(u)}= \begin{cases}\left(\left|x_{(u)}\right|^{2}+\left|\hat{x}_{(u)}\right|^{2}\right) & n_{t}^{(u)} \neq \hat{n}_{t}^{(u)} \\ \left(\left|x_{(u)}-\hat{x}_{(u)}\right|^{2}\right) & n_{t}^{(u)}=\hat{n}_{t}^{(u)} \\ 0 & n_{t}^{(u)}=\hat{n}_{t}^{(u)} \text { and } x_{(u)}=\hat{x}_{(u)} .\end{cases}
$$

Note that (42) presents an analytical treatment of the most general case of SM using variable amplitude modulation for the signal symbol. Given this, the system using the interference-aware detector behaves in a similar fashion to the noise-limited system, in that for an arbitrarily high SNR, each user can achieve an arbitrarily low ABER. It should be pointed out that due to the simultaneous detection process, the users with the best SNR will not be able to achieve their single-user-lower-bound (SULB).
The exact effect of the additional nodes/users is further discussed in the "Simulation results and discussion" section.

\section{Simulation results and discussion}

In this section, we aim to show the performance of the interference-unaware and interference-aware detectors proposed in (2) and (41). In particular, we aim to show that (41) can successfully decode the incoming streams for all nodes. Numerical results demonstrate that (12) and (42) provide tight upper bounds for the ABER of the detectors at high SNR in the interferencelimited scenario. Furthermore, we demonstrate that the interference-aware detector for SM performs better than the ML detector for a multi-user SIMO system using QAM.

The proposed interference-aware detector is jointly optimal for all nodes and does not suffer from the nearfar problem, but it needs full channel state information (CSI) from all possible transmitting antennas to each receiving antenna. In addition, finding the optimal solution is an exponentially complex problem. Assuming each node has the same number of transmit antennas, $N_{t}$, and uses the same signal constellation with $M$ points, then the interference-aware ML detector proposed has $\mathcal{O}\left(\left(M N_{t}\right)^{N_{u}}\right)$ computational complexity which is proven to be NP-complete [33].

To justify our $\mathcal{O}(\cdot)$ complexity, we point to the key difference between SM and other multi-user MIMO schemes, the signal and spatial domains combine to form a single SM symbol. The constellation size, i.e. the spectral efficiency of any SM system, depends on the multiplication of the number of available transmit antennas and the signalsymbol constellation used, $M N_{t}$. This is in stark contrast to other MIMO systems where each spatial branch is used to increase the diversity or multiplexing gains. In such a system, if each transmit antenna is used for multiplexing gains, the system has a maximum spectral efficiency of $M^{N_{t}}$. From here, the detection complexity of a single user SM system is given by $\mathcal{O}\left(M N_{t}\right)$, while the detection complexity of a single user MIMO system used for multiplexing gains is given by $\mathcal{O}\left(M^{N_{t}}\right)$. In this case, the two systems have different spectral efficiencies. Alternatively, if the two systems operate at the same spectral efficiency, then their complexities will be of the same $\mathcal{O}$ order, but 
the cost, in terms of RF chains and power consumption would not be. The aim of this study is to characterise the behaviour of SM in the multi-user, interference-limited scenario and compare it to the complexity and cost equivalent multi-user MIMO system. As discussed in the "Introduction" section and given the complexity expressions for the single user MIMO system and the single user SM system, it is apparent that the only valid complexity and cost equivalent comparison is to compare multi-user SM with multi-user SIMO. The optimal ML detector for the interference-aware SIMO system has $\mathcal{O}\left((\tilde{M})^{N_{u}}\right)$ computational complexity, where $\tilde{M}=M N_{t}$ from the "SIMO system utilising QAM (noise-limited scenario)" section, making it comparable to the interference-aware SM detector. Recent work on sphere detection algorithms may be used to alleviate this computational cost [9]. Despite the generality of our results, we restrict our simulation results to two and three node scenarios for the sake of conciseness.

\section{Simulation setup}

A frequency-flat Rayleigh fading channel with no correlation between the transmitting antennas and AWGN is assumed. Perfect CSI is assumed at the receiving node, with no CSI at the transmitter. Only one of the available transmit antennas for each node is active at any transmitting instance. In theory, each node independently decides the number of transmit antennas, and, by extension, the signal-symbol modulation it uses. In the simulation each node has the same number of transmit antennas as well as the same spectral efficiency target. In each of the figures, there are three sets of results presented: (i) the simulation results for the multi-user detector for each node, denoted by $\operatorname{Sim}\left(\mathrm{N}_{(\xi)}\right)$, (ii) the theoretical results from (12) or (42) for the node of interest and (iii) the SULB, denoted by SULB $\left(\mathrm{N}_{(\xi)}\right)$. We define the asymptotically tight SULB as the system performance in a noise-limited scenario given in (26) which is governed purely by its SNR, defined as $E_{m} / N_{o}$. It should be noted that in Figures 2, 3 and 4 in addition to (26), ASER/2, i.e. (28) divided by 2 , is presented and overlaps (26); both are denoted as $\operatorname{SULB}\left(\mathrm{N}_{(\xi)}\right)$ in Figures 2,3 and 4 . This serves to justify the use of (28) in our asymptotic analysis in the "Asymptotic analysis of an interference-unaware detector" section. In addition, (26) is based on the union bound technique and, in some results, $\operatorname{SULB}\left(\mathrm{N}_{(\xi)}\right)$ is above 1, which is

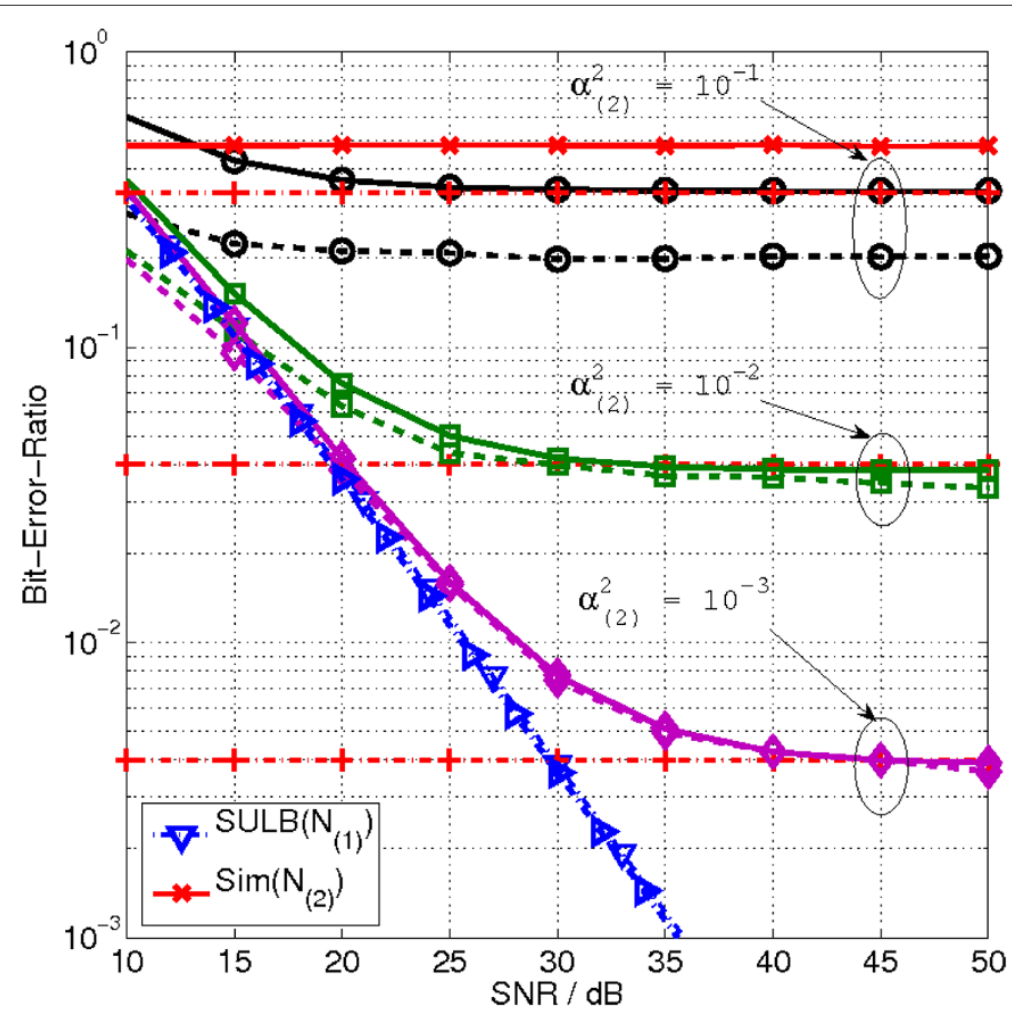

Figure $2 \boldsymbol{N}_{\boldsymbol{t}}^{(\boldsymbol{u})}=\mathbf{4}, \boldsymbol{N}_{\boldsymbol{r}}=\mathbf{1}$ and a spectral efficiency of $\mathbf{4} \mathrm{bits} / \mathrm{s} / \mathrm{Hz}$. ABER for node 1 using the interference-unaware detector with $\alpha_{(1)}^{2}=1$ and node 2 with a varying $\alpha_{(2)}^{2}$. Dashed lines denote simulation results for node 1 while solid lines denote the analytical upper bound. Since the analytical bound is asymptotically tight, at low ABER the dashed and solid lines overlap. Each constant value dashed-dot line with ' + ' markers corresponds to the asymptote derived in (34) using (40) for the different values of $\alpha_{(2)}^{2}$. 


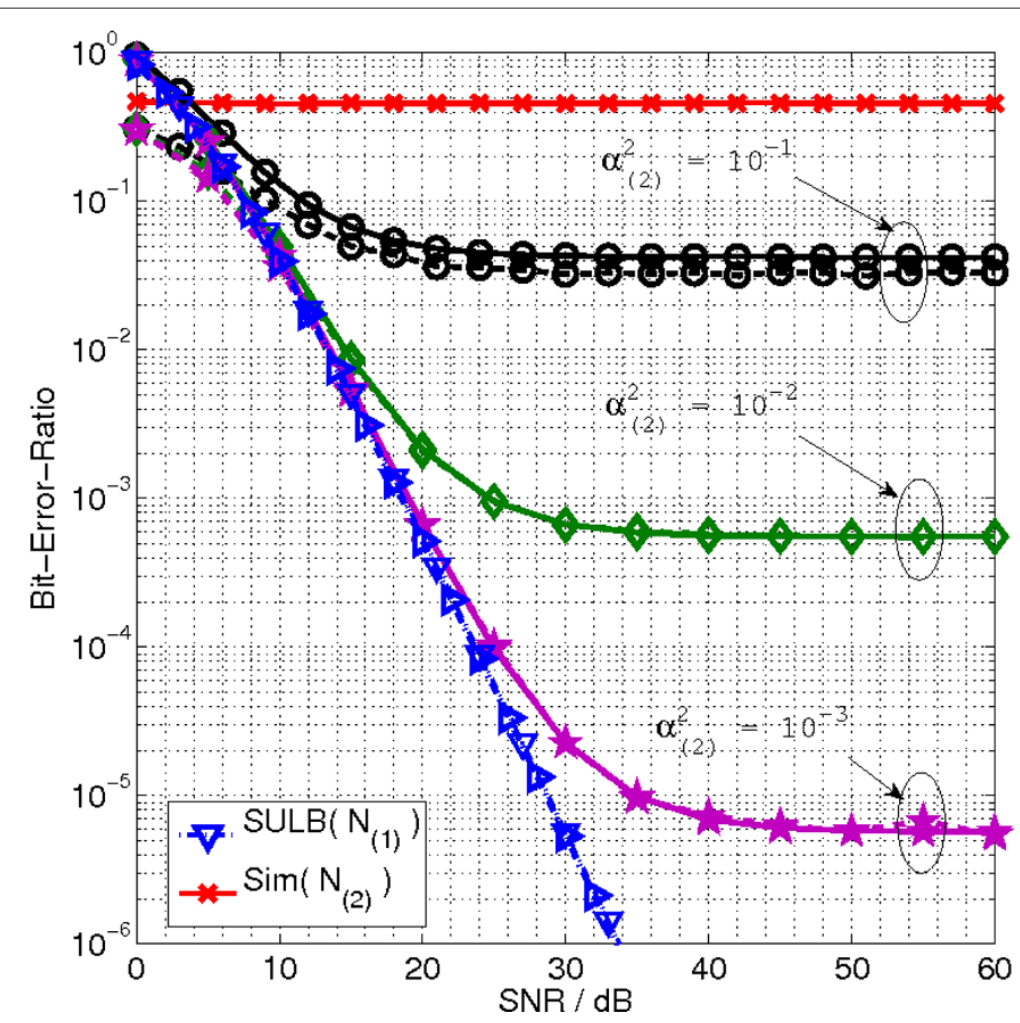

Figure $3 \boldsymbol{N}_{\boldsymbol{t}}^{(\boldsymbol{u})}=\mathbf{4}, \boldsymbol{N}_{\boldsymbol{r}}=\mathbf{2}$ and a spectral efficiency of $\mathbf{4} \mathrm{bits} / \mathbf{s} / \mathbf{H z}$. ABER for node 1 using the interference-unaware detector with $\alpha_{(1)}^{2}=1$ and node 2 with a varying $\alpha_{(2)}^{2}$. Dashed lines denote simulation results for node 1 while solid lines denote the analytical upper bound. Since the analytical bound is asymptotically tight, at low ABER the dashed and solid lines overlap.

impossible for a real system. In this regard, the SULB is a lower bound on the analytical performance of each system only at low ABER. To help illustrate the difference in the behaviour of the two detectors, the channel attenuations, $\alpha_{(u)}^{2}$, are set in 10-dB intervals. In general, $\alpha_{(u)}^{2}$ may be any real number. Throughout the results, QAM modulation is used for the signal-symbol modulation in SM with the notable exception of Figure 2, where PSK modulation is used to illustrate the accuracy of work done in the "Asymptotic analysis of an interference-unawaredetector" section.

\section{Results for interference-unaware detection}

Asymptotic results in the "Asymptotic analysis of an interference-unaware detector" section, in particular (34) using QPSK for the signal-symbol modulation are verified in Figure 2. In this case, $\Psi$ is strictly defined by (22). The horizontal lines in Figure 2 represent (34) for varying values of $\alpha_{(u)}^{2}$ using QPSK modulation. From Figures 3, 4 and 5, where QAM is used for the signal-symbol modulation, the developed analytical framework is accurate in all the presented instances. By moving from Figure 3 to Figure 3, where an additional receive antenna is added, the analytical model presented in (12) is a tight upper bound on the system in the high SINR region. As the channel attenuations for the interfering nodes increase, the detector approaches SULB. Similar to the effects observed in Figures 2 and 3, Figure 4 shows how the tightness of the bound improves as the number of receive antennas increases. In all instances where the interference-unaware detector is used, the node with the strongest SNR dominates the detection. The bit streams of all other nodes are not decoded. Since the SINR does not change, all other nodes remain below the effective noise floor at the receiver. This is apparent by looking at the simulation results for $N_{(2)}$ and $N_{(3)}$ in Figures 2, 3 and 4. By looking at the presented results, we conclude that (34) tightens as the system approaches its ideal transmission and the mass of the complex Gaussian distributions around each SM constellation point concentrates around the mean. This is achieved by decreasing the interference in the system or by increasing the number of receive antennas.

Figure 4 shows that the increase in diversity resulting from the addition of only a single receive antenna significantly influences the system performance. The addition of the receive antenna increases the Euclidean distance between the received and hypothesis vectors, 


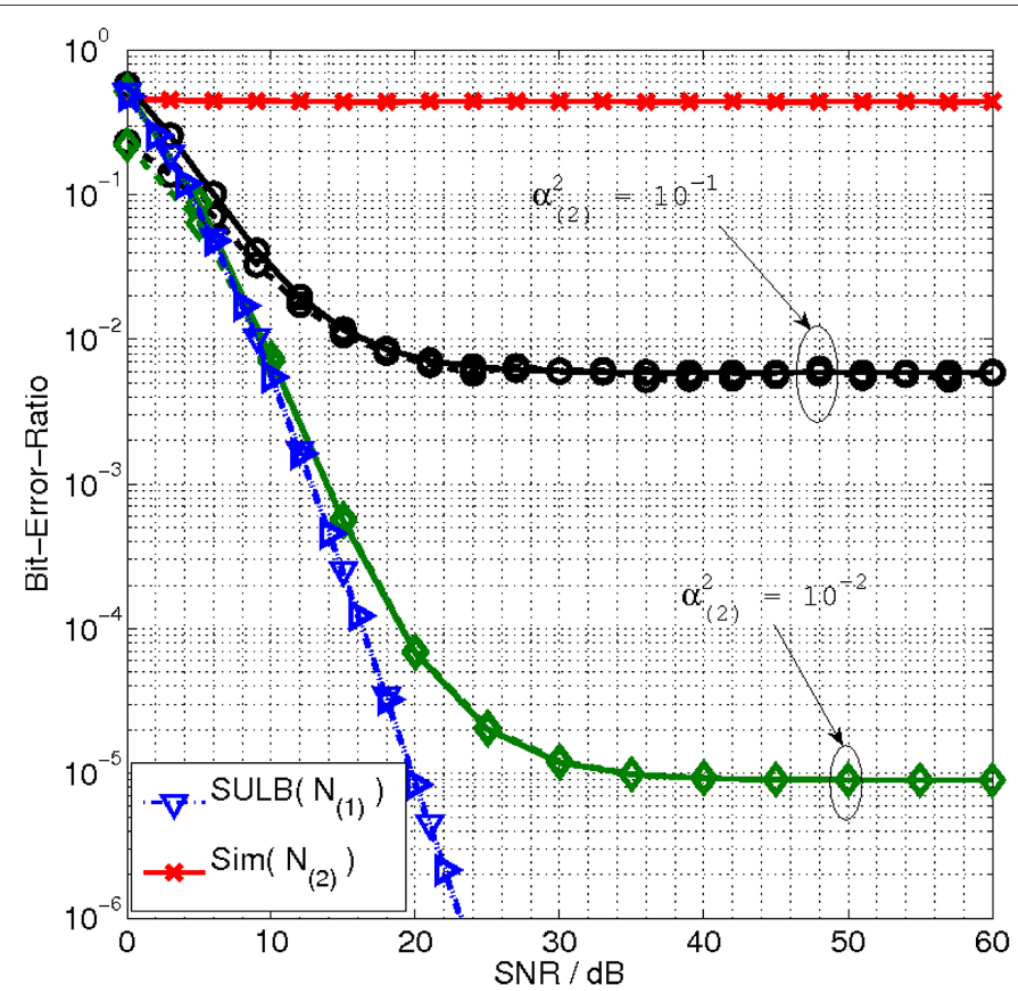

Figure $4 \boldsymbol{N}_{\boldsymbol{t}}^{(\boldsymbol{u})}=\mathbf{4}, \boldsymbol{N}_{\boldsymbol{r}}=\mathbf{3}$ and a spectral efficiency of $\mathbf{4} \mathrm{bits} / \mathrm{s} / \mathrm{Hz}$. ABER for node 1 using the interference-unaware detector with $\alpha_{(1)}^{2}=1$ and node 2 with a varying $\alpha_{(2)}^{2}$. Dashed lines denote simulation results for node 1 while solid lines denote the analytical upper bound. Since the analytical bound is asymptotically tight, at low ABER the dashed and solid lines overlap.

which results in a lower ABER. Comparing Figure 2 with Figure 3 and similarly Figure 3 with Figure 4 where the number of receive antennas is increased in each figure, showing how the addition of a single receive antenna is equivalent to lowering the interference $\left(\alpha_{(u)}^{2}\right)$ by more than $10 \mathrm{~dB}$. In fact, the effect of each receive antenna is more pronounced as the imbalance between the desired and interfering links increases. By looking at the results for $\alpha_{(2)}^{2}=10^{-2}$ in Figure 2, the results for $\alpha_{(2)}^{2}=10^{-2}$ in Figure 3, and the results for $\alpha_{(2)}^{2}=10^{-2}$ in Figure 4 at an SNR of $40 \mathrm{~dB}$, we see the error rate of the simulation and analytical prediction moving from $2 \times 10^{-1}$ in Figure 2, to $4 \times 10^{-3}$ in Figure 3, to $9 \times 10^{-5}$ in Figure 4. This ABER decrease shows how the number of receive antennas dominates the performance of SM in general, and particularly in an interference limited scenario. Figure 5 demonstrates that the findings can be extended even in the presence of two interfering nodes.

From the presented results it is clear that when the interference-unaware detector is used, the system ABER plateaus at the derived limits, irrespective of the transmit power being used. As discussed in the "Interferenceunaware detection" section, and as work in [20] has shown, the ABER improves when the number of transmit or receive antennas is increased, i.e. the system achieves coding gains.

\section{Results for interference-aware detection}

In Figure 6 we see the performance of the jointly optimal interference-aware ML detector for a two user scenario. Figure 6 clearly demonstrates that the analytical model presented in (42) represents an asymptotically tight upper bound for the system in the high SNR region. The node with the worse channel attenuation performs close to its SULB. This is not the case for the node with the better channel.

To understand this, we can think of the multi-user ML detector as employing interference cancellation for the node with the worse channel attenuation. If the interfering user is sufficiently powerful, then the primary source of errors for the weakest node is the background AWGN rather than the randomness caused by the interfering signal [29]. All users that have good channel conditions can be considered as strong interferers, so when they are removed, the weakest nodes obtain performance closer to their SULB, i.e. the interference-aware detector is akin to strong interference cancellation for the weakest node. On the contrary, for the nodes with better channel conditions, the primary source of errors is the randomness caused by 


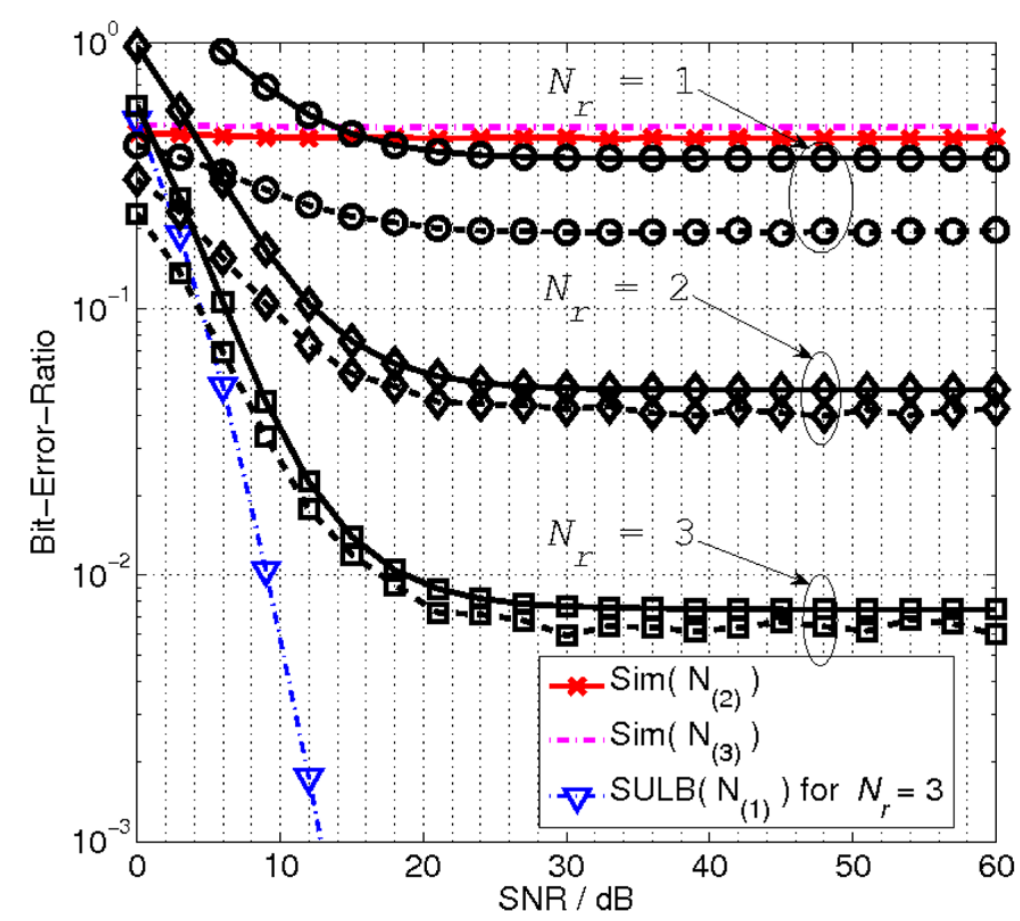

Figure $5 N_{\boldsymbol{t}}^{(\boldsymbol{u})}=\mathbf{4}$, a varying $\boldsymbol{N}_{\boldsymbol{r}}$ and a spectral efficiency of $\mathbf{4} \mathrm{bits} / \mathrm{s} / \mathrm{Hz}$. ABER for node 1 using the interference-unaware detector with $\alpha_{(1)}^{2}=1$, node 2 with $\alpha_{(2)}^{2}=0.1$ and node 3 with $\alpha_{(3)}^{2}=0.01$. All presented curves are for node 1 unless otherwise stated in the legend. Dashed lines denote simulation results for node 1 while solid lines denote the analytical upper bound. We note that the addition of more receive antennas reduces the ABER and hence closes the gap between the analytical and simulation results.

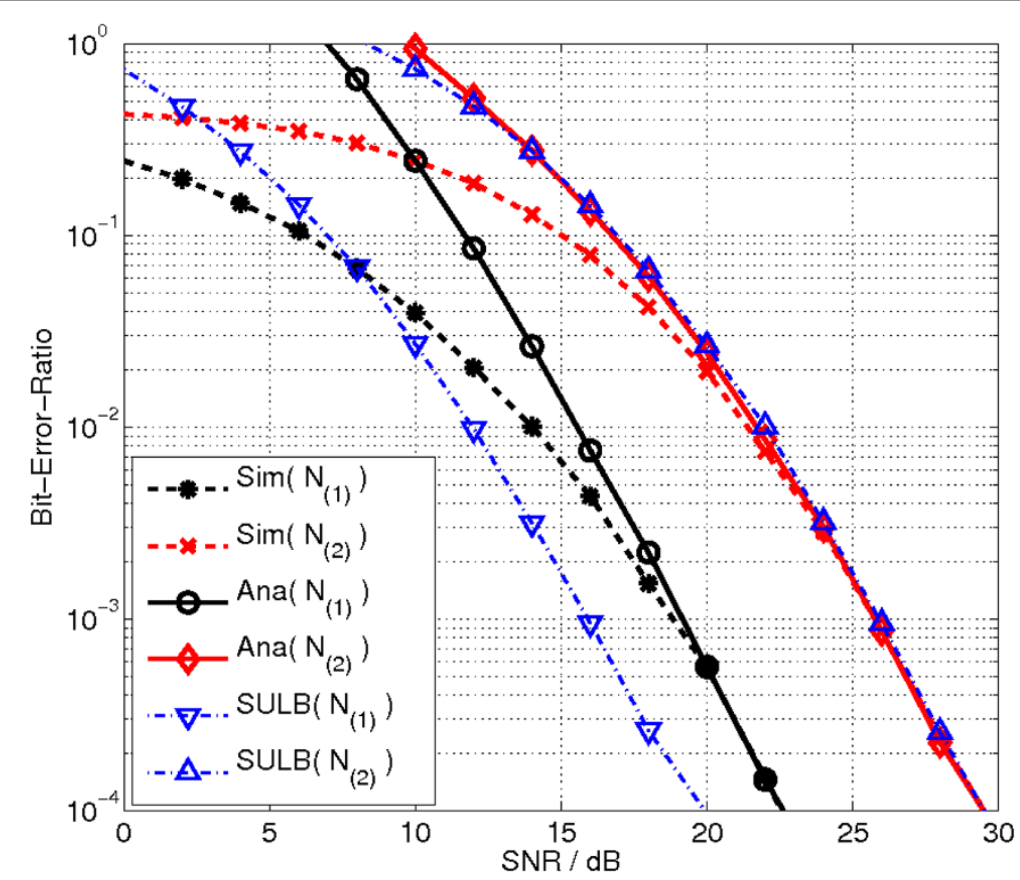

Figure $\mathbf{6} \boldsymbol{N}_{\boldsymbol{t}}^{(\boldsymbol{u})}=\mathbf{2}, \boldsymbol{N}_{\boldsymbol{r}}=\mathbf{3}$ and a spectral efficiency of $\mathbf{4} \mathrm{bits} / \mathrm{s} / \mathrm{Hz}$. ABER using the interference-aware detector for node 1 with $\alpha_{(1)}^{2}=1$ and node 2 with $\alpha_{(2)}^{2}=0.1$. Ana $\left(\mathrm{N}_{(u)}\right)$ denotes the analytical upper bound for node $u$. Since the analytical bound is asymptotically tight, at low ABER the dashed and solid lines overlap. 


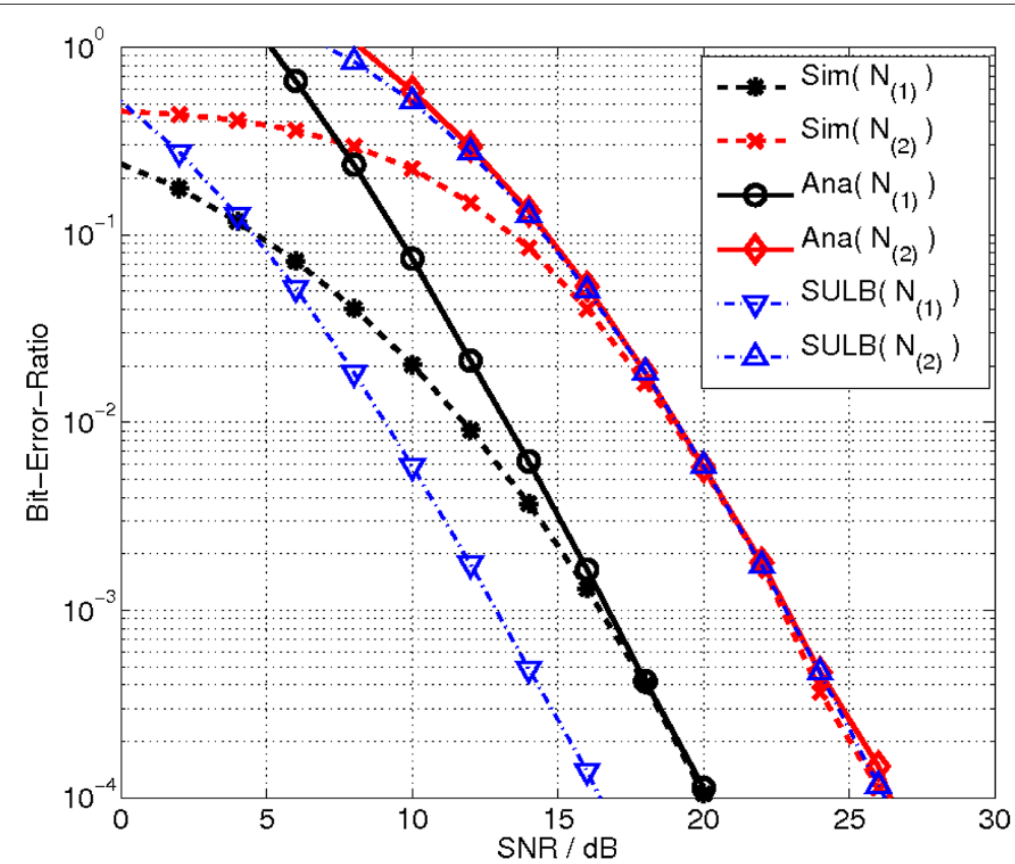

Figure $7 N_{t}^{(u)}=\mathbf{2}, \boldsymbol{N}_{\boldsymbol{r}}=\mathbf{3}$ and a spectral efficiency of $\mathbf{4} \mathrm{bits} / \mathrm{s} / \mathrm{Hz}$. ABER using the interference-aware detector for node 1 with $\alpha_{(1)}^{2}=1$ and node 2 with $\alpha_{(2)}^{2}=0.1$. Ana $\left(\mathrm{N}_{(u)}\right)$ denotes the analytical upper bound for node $u$. Since the analytical bound is asymptotically tight, at low ABER the dashed and solid lines overlap.

the interfering signal rather than the background AWGN, which is why the nodes with better channel conditions never perform near their SULB.

The addition of more transmit antennas at each of the nodes results in coding gains for each node as can be seen when Figures 6 and 7 are compared. The reduction in ABER as the number of transmit antennas increases is explained by realising that as the number of transmit antennas increases, the average variance $\sigma_{z}^{2}$ increases. As $\sigma_{z}^{2}$ increases, it leads to a larger Euclidean distance in (10) and (43). The Euclidean distance is increased because there are more cases where the variance is the summation of the individual symbol constellation points, rather than the difference, i.e. $n_{t}^{(\xi)}=\hat{n}_{t}^{(\xi)}$ occurs less frequently. Effectively, more transmit antennas mean that the transmit vectors are spread in a larger Euclidean space. This effect can only be observed when the same spectral efficiency is maintained. In particular, a 2- $\mathrm{dB}$ coding gain is apparent when comparing Figures 6 and 7 at an ABER of $10^{-4}$. However, increasing the number of transmit antennas does not change the relative behaviour of the system, i.e. the SNR difference between the ABER curves of the two nodes remains constant. This behaviour is expected when we consider that (17) is independent of $N_{t}$ and influenced only by $N_{r}$.

Figure 8 shows the performance of the system when the number of receive antennas is increased. On the one hand, moving from Figures 7 to 8 , for a fixed spectral efficiency and a fixed number of transmit antennas, the addition of more receive antennas results in an increasing gap between the analytical ABER curves of the two nodes. In particular, a gap of $4 \mathrm{~dB}$ between the performance of nodes 1 and 2 with $N_{r}=2$ is increased to around $7 \mathrm{~dB}$ when $N_{r}=4$ and further increased to $9 \mathrm{~dB}$ for $N_{r}=8$. On the other hand, given that the two nodes experience a channel gain difference of $10 \mathrm{~dB}$, we know that the interference-aware detector cannot reach the performance of independent detection and the SULB for the node with the better channel attenuation. Nonetheless, the gap between their respective ABER curves tends toward the difference between their respective channel attenuation as $N_{r}$ grows.

These trends can also be observed if we look at the progression of the ABER curves in Figures 9 and 10. Figures 9 and 10 show the system performance of three users and varying $N_{r}$. Similar to the two user scenario, as $N_{r}$ increases, for the three user case, each user performs better and slowly closes the gap to its SULB. As expected, the addition of more nodes increases the interference and pushes the performance of each node further from its SULB, noticeable when comparing Figures 7 and 10 for node 2 .

Lastly, Figure 11 demonstrates that in the multiple access interference-limited scenario, by using the interference-aware detector, SM performs better than the complexity and cost equivalent ML detector for 


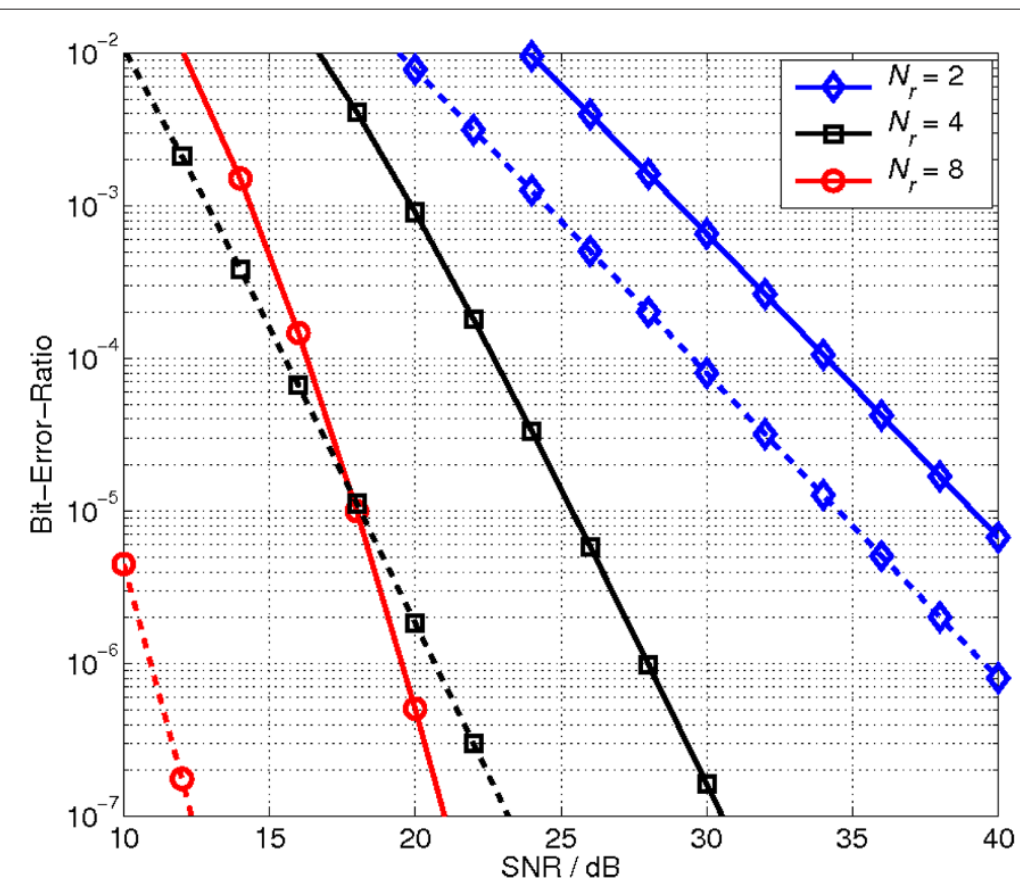

Figure $8 \boldsymbol{N}_{\boldsymbol{t}}^{(\boldsymbol{u})}=\mathbf{4}$, a varying $\boldsymbol{N}_{\boldsymbol{r}}$ and a spectral efficiency of $\mathbf{4} \mathrm{bits} / \mathrm{s} / \mathrm{Hz}$. ABER using the interference-aware detector for node 1 with $\alpha_{(1)}^{2}=1$ and node 2 with $\alpha_{(2)}^{2}=0.1$. Dashed lines denote the performance of node 1 with a varying number of receive antennas while solid lines denote the performance of node 2 with a varying number of receive antennas.

the multi-user SIMO system. Specifically, SM exhibits an approximately $3 \mathrm{~dB}$ better performance in terms of SNR at an ABER of $10^{-4}$ for each user. The relatively constant coding gain is the result of the similar detection used for both systems. While multi-user SM and multi-user SIMO may be comparable in terms of detector complexity and the number of transmit and receive RF chains required, each SM node requires multiple transmit antenna elements. The multiple transmit antennas mean that the SM constellation points are spread in a larger Euclidean space and thus have lower error probability.

\section{Summary and conclusions}

In this study, the performance of SM in the multiple access, interference-limited scenario was investigated. Two ML detectors for use with SM were discussed.

The interference-unaware detector was defined and studied in the limit as the SNR approached infinity. Its performance over uncorrelated Rayleigh fading channels was studied and a closed form solution for the upper bound of the system was provided. It was shown that this detector inevitably reaches an error floor which is dependent on the system SINR. The exact level was defined as a function and concrete examples were provided. It was shown that the increase in the number of receive antennas has a greater impact on the asymptotic performance of the system compared to reducing the interference in the system. The addition of a single receive antenna resulted in greater SNR gains than reducing the interference, $\alpha_{(u)}^{2}$, by more than $10 \mathrm{~dB}$ at high SNR. This implies that the number of receive antennas dominates the performance of SM in general, and particularly in an interference-limited scenario.

The interference-aware ML detector for SM was proposed. As with the interference-unaware detector, its performance over uncorrelated Rayleigh fading channels was studied and a closed form solution for the upper bound of the system was provided. In addition to avoiding the error floor present in the interferenceunaware detector, the jointly optimal detector results in a noise-limited scenario for the detection of all transmitted streams, i.e. an arbitrarily small ABER can be obtained by any user for a sufficiently high SNR. On the one hand, for the same spectral efficiency, increasing the number of transmit antennas at each of the nodes from 2 to 4 resulted in SNR gains of around $2 \mathrm{~dB}$. This measure did not, however, have any effect on the coding gain difference between the ABER curves. On the other hand, increasing the number of receive antennas increased the diversity of the system. This, increased the coding gain difference between the ABER curves of the nodes because the receiver could distinguish the channels more easily and better mitigate 
interference. The impact on the diversity and coding gains further shows the importance of the number of receive antennas in any SM system. A limiting factor, as with all ML detectors, is the complexity. In addition, the receiver must have channel knowledge from all transmitting nodes. These two limitations constrain the application of this detector to the uplink scenario. The interference-aware detector enabled SM to perform better in terms of ABER than the complexity and cost equivalent multi-user SIMO system in an interference-limited environment.

This study demonstrated that in order to effectively apply SM in an interference-limited scenario, the number of receive antennas should be maximised. Although more computationally complex than the interference-unaware detector, the interference-aware detector can guarantee that the system does not reach an error floor.

\section{Appendix}

Derivation of (32)

We begin by considering $\widetilde{\psi_{1}}$ which corresponds to $\sigma_{z}^{2}=$ $\left|x^{(\xi)}\right|^{2}+\left|\hat{x}^{(\xi)}\right|^{2}$. For this event, we seek to bound $\sigma_{z}^{2}$ to two which implies that we should consider

$$
\left(d_{\epsilon}^{2}+d_{\hat{\epsilon}}^{2}\right) g_{\mathrm{QAM}} \leq 2
$$

where $\epsilon \in\{(1,1),(2,1),(3,1)\}$ and $d_{\epsilon}$ are the distances defined in Figure 12 and $g_{\mathrm{QAM}}=\frac{3}{2\left(M^{(\xi)}-1\right)}$. We introduce the normalising factor $g_{\mathrm{QAM}}$, given in [32], to maintain unity power in our constellation. We see that there are only four possible combinations that satisfy (46). In particular

- $\left(d_{(1,1)}^{2}+d_{(1,1)}^{2}\right) g_{\mathrm{QAM}}=0.4$, occurs $\left(M^{(\xi)}\right)^{2} / 8=32$ times,

- $\left(d_{(1,1)}^{2}+d_{(2,1)}^{2}\right) g_{\mathrm{QAM}}=1.2$, occurs $\left(M^{(\xi)}\right)^{2} / 4=64$ times,

- $\left(d_{(1,1)}^{2}+d_{(3,1)}^{2}\right) g_{\mathrm{QAM}}=2$, occurs $\left(M^{(\xi)}\right)^{2} / 8=32$ times and

- $\left(d_{(2,1)}^{2}+d_{(2,1)}^{2}\right) g_{\mathrm{QAM}}=2$, occurs $\left(M^{(\xi)}\right)^{2} / 2=128$ times.

We know that these combinations occur for every instance when $n_{t} \neq \hat{n}_{t}$. For a system with $N_{t}^{(\xi)}$ transmit antennas, we have $\left(\begin{array}{c}N_{t}^{(\xi)} \\ 2\end{array}\right)$ unique pairings. This leads to

$$
\widetilde{\psi_{1}}=\left((0.4 / 2)^{-N_{r}} 32+(1.2 / 2)^{-N_{r}} 64+32+128\right)\left(\begin{array}{c}
N_{t}^{(\xi)} \\
2
\end{array}\right) \text {. }
$$

Having established the derivation of $\widetilde{\psi_{1}}$, we turn our attention to $\widetilde{\psi_{2}}$ which corresponds to $\sigma_{z}^{2}=\left|x^{(\xi)}-\hat{x}^{(\xi)}\right|^{2}$. If we look at Figure 12, we see there are additional combinations aside from the ones denoted. To this extent, our

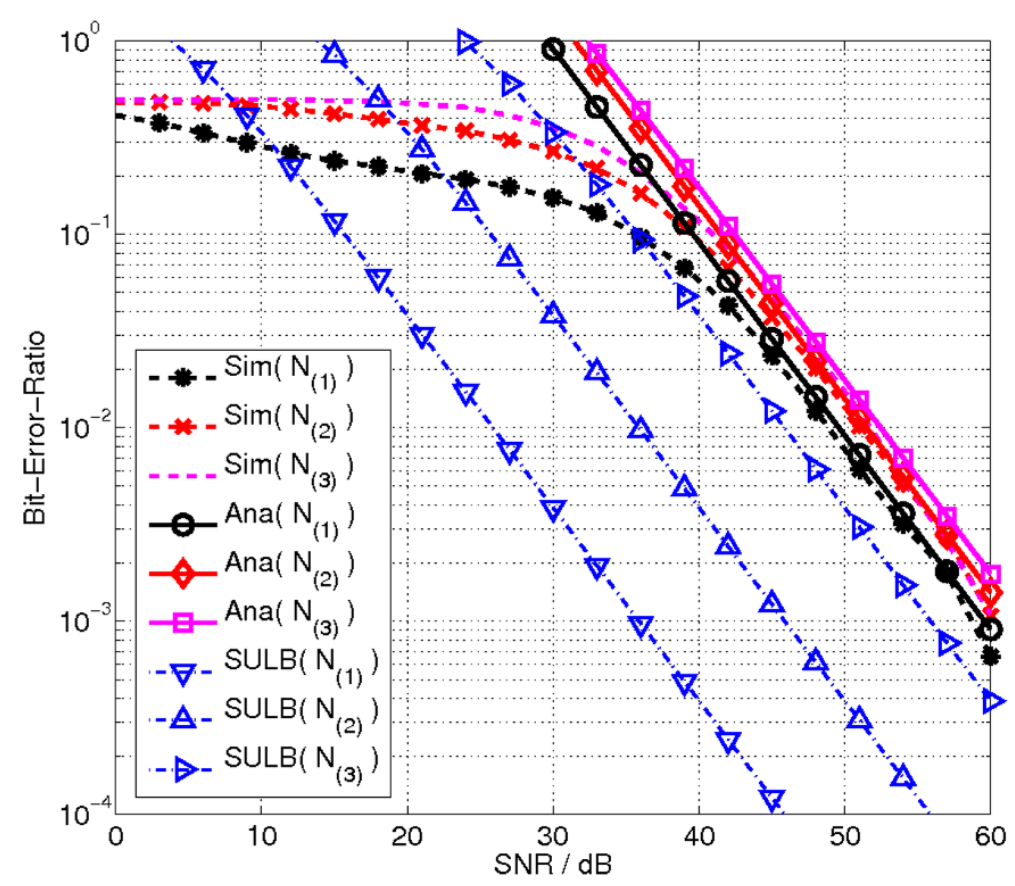

Figure $9 \boldsymbol{N}_{\boldsymbol{t}}^{(\boldsymbol{u})}=\mathbf{4}, \boldsymbol{N}_{\boldsymbol{r}}=\mathbf{1}$ and a spectral efficiency of $\mathbf{4} \mathbf{b i t s} / \mathbf{s} / \mathbf{H z}$. ABER for node 1 with $\alpha_{(1)}^{2}=1$, node 2 with $\alpha_{(2)}^{2}=0.1$ and node 3 with $\alpha_{(3)}^{2}=0.01$. Ana $\left(\mathrm{N}_{(u)}\right)$ denotes the analytical upper bound for node $u$. Since the analytical bound is asymptotically tight, at low ABER the dashed and solid lines overlap. 


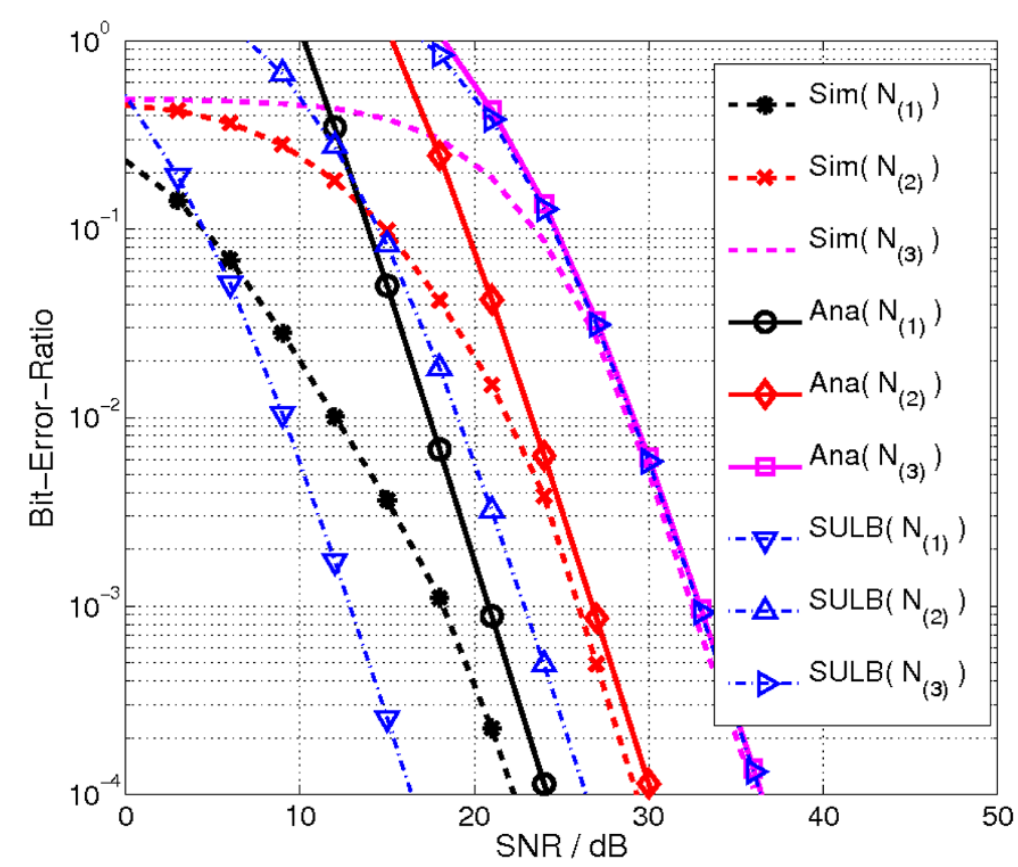

Figure $10 \boldsymbol{N}_{\boldsymbol{t}}^{(\boldsymbol{u})}=\mathbf{4}, \boldsymbol{N}_{\boldsymbol{r}}=\mathbf{3}$ and a spectral efficiency of $\mathbf{4} \mathbf{b i t s} / \mathbf{s} / \mathbf{H z}$. ABER for node 1 with $\boldsymbol{\alpha}_{(1)}^{2}=1$, node 2 with $\boldsymbol{\alpha}_{(2)}^{2}=0.1$ and node 3 with $\alpha_{(3)}^{2}=0.01$. Ana $\left(\mathrm{N}_{(u)}\right)$ denotes the analytical upper bound for node $u$. The analytical bound is again shown to be asymptotically tight at low ABER.

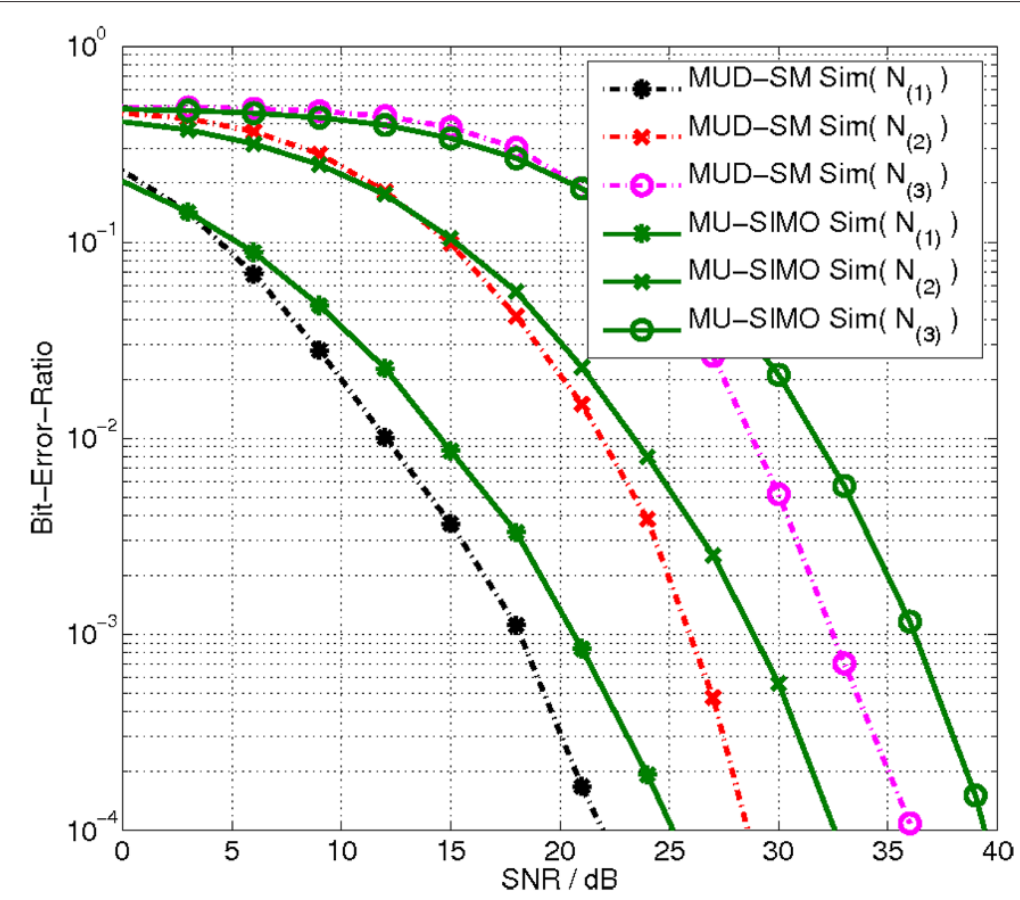

Figure $11 N_{t}^{(u)}=\mathbf{4}, N_{r}=\mathbf{3}$ and a spectral efficiency of $\mathbf{4} \mathrm{bits} / \mathrm{s} / \mathrm{Hz}$. ABER of multi-user SM and multi-user SIMO using interference-aware detectors with node 1 experiencing channel attenuation of $\alpha_{(1)}^{2}=1$, node 2 with $\alpha_{(2)}^{2}=0.1$ and node 3 with $\alpha_{(3)}^{2}=0.01$. 
bound, $\sigma_{z}^{2}=\min \left\{\sigma_{z}^{2}, 2\right\}$, serves to simplify the counting and set many of them to 2 . In particular, we see that when $\sigma_{z}^{2}=d_{(5,2)}^{2}$, then $\sigma_{z}^{2}>2$. In this case, we bound $\sigma_{z}^{2}$ to 2 and realise that $d_{(4,2)}$ is the largest distance that we must account for in our expectation analysis. We note that our approach is applicable to larger constellation sizes and, after some careful counting, we see that in general for a given constellation size of $M^{(\xi)}$ we have that

- $d_{(1,2)}$ occurs a total of $D^{(1)}=4 \sqrt{M^{(\xi)}}\left(\sqrt{M^{(\xi)}}-1\right)$ times,

- $d_{(2,2)}$ occurs a total of $D^{(2)}=4 \sqrt{M^{(\xi)}}\left(\sqrt{M^{(\xi)}}-2\right)$ times,

- $d_{(3,2)}$ occurs a total of $D^{(3)}=4\left(\sqrt{M^{(\xi)}}-1\right)\left(\sqrt{M^{(\xi)}}-1\right)$ times and,

- $d_{(4,2)}$ occurs a total of $D^{(4)}=8\left(\sqrt{M^{(\xi)}}-1\right)\left(\sqrt{M^{(\xi)}}-2\right)$ times. 16:

By looking at Figure 12, we can easily see that for $M^{(\xi)}=$

- $d_{(1,2)}^{2} g_{\mathrm{QAM}}=0.4$, occurs exactly 48 times,

- $d_{(2,2)}^{2} g_{\mathrm{QAM}}=1.6$, occurs exactly 32 times,

- $d_{(3,2)}^{2} g_{\mathrm{QAM}}=0.8$, occurs exactly 36 times and

- $d_{(4,2)}^{2} g_{\mathrm{QAM}}=2$, occurs exactly 48 times.

We realise that the combinations counted above are applicable for every transmit antenna. This implies that we must multiply each count by $N_{t}^{(\xi)}$ which leads to

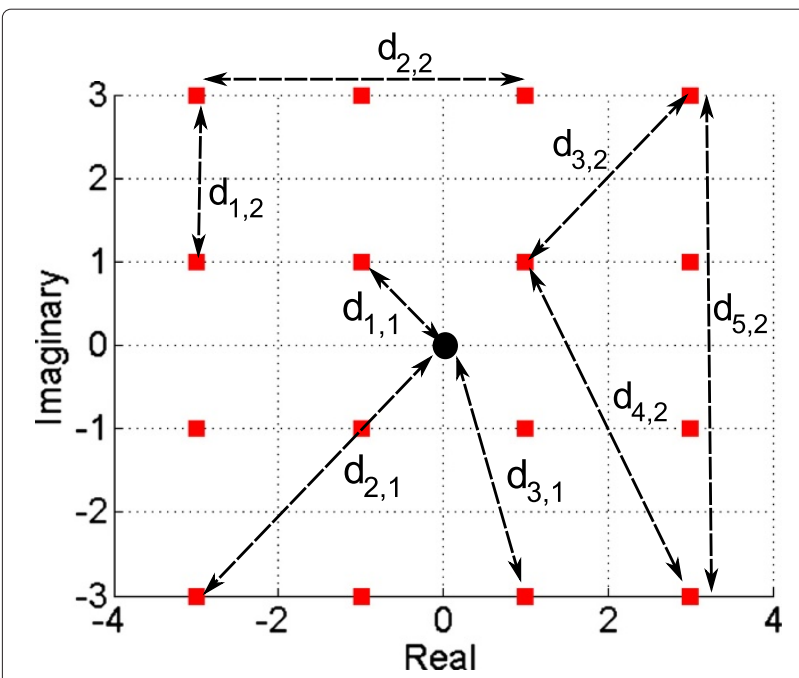

Figure 12 The red squares denote 16-QAM constellation points. In this image, the constellation is not normalised. The figure shows possible distance combinations between each of the constellation points and their distances to the origin.

$$
\begin{gathered}
\widetilde{\psi_{2}=}\left((0.4 / 2)^{-N_{r}} 48+(1.6 / 2)^{-N_{r}} 32\right. \\
\left.+(1.6 / 2)^{-N_{r}} 36+48\right) N_{t}^{(\xi)} .
\end{gathered}
$$

To arrive at a final solution, we set all other combination values to $g_{\mathrm{QAM}}$ as we enforce our bound on $\sigma_{z}^{2}$ giving

$$
\begin{aligned}
D_{\text {ones }}= & \left(M^{(\xi)} N_{t}^{(\xi)}\right)^{2}-\left(M^{(\xi)}\right)^{2}\left(\begin{array}{c}
N_{t}^{(\xi)} \\
2
\end{array}\right) \\
& -\left(D^{(1)}+D^{(2)}+D^{(3)}+D^{(4)}\right)^{2} N_{t}^{(\xi)}
\end{aligned}
$$

which is the number of elements for which $\sigma_{z}^{2}$ was set to 2 , i.e. $\left(\sigma_{z}^{2} / 2\right)^{-N_{r}}=1$. We now average and arrive at $\Psi$. To achieve this, we merely normalise our sum by $\left(M^{(\xi)} N_{t}^{(\xi)}\right)^{2}$ to account for the number of possible events, resulting in

$$
\Psi=\frac{\widetilde{\psi_{1}}+\widetilde{\psi_{2}}+D_{\text {ones }}}{\left(M^{(\xi)} N_{t}^{(\xi)}\right)^{2}}
$$

which when simplified becomes (32).

In Figure 13 we compare the asymptote of SM, given by (34), in the noise-limited scenario for $M^{(\xi)}=16$ with and without using (32). Figure 13 exemplifies the accuracy and tightness of our approach.

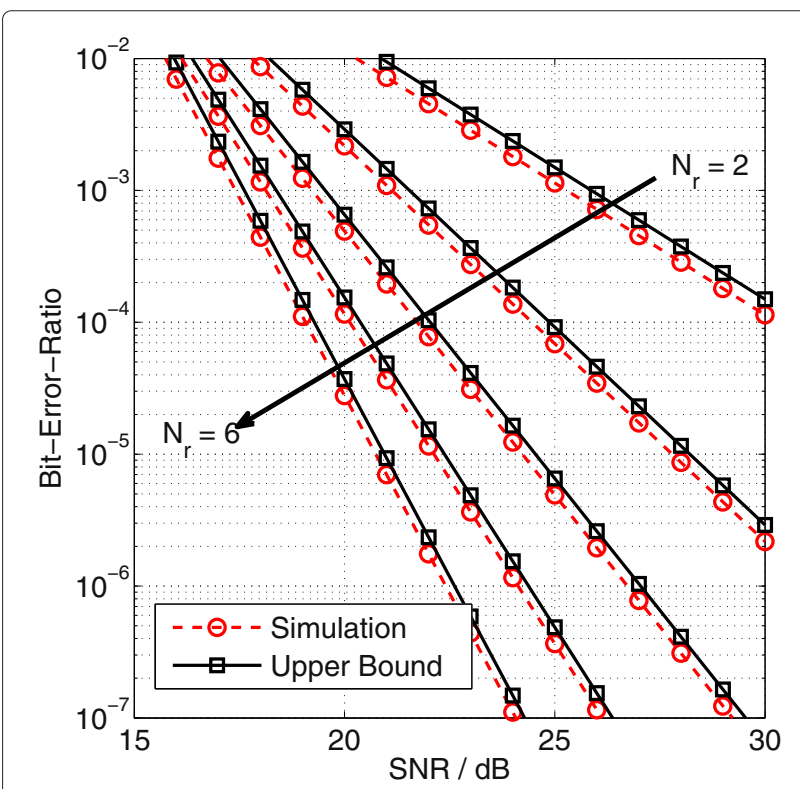

Figure 13 The dashed lines with circle markers denote the asymptote for the system if the expectation for $\left(\sigma_{z}^{2} / 2\right)^{-N_{r}}$ is obtained via simulations. The solid lines with square markers denote the asymptote when (32) is used. Moving from the rightmost to the leftmost pairs of curves, each pair corresponds to an incremental increase in $N_{r}$ from 2 to 6 . The system is using two transmit antennas and has an overall spectral efficiency of 5 bits $/ \mathrm{s} / \mathrm{Hz}$. 
Reaching (38) from (34) with (31) and (37)

Combining (34) with (31) results in

$$
\begin{aligned}
\lim _{\gamma_{\mathbf{I}} \rightarrow \infty} \frac{\mathrm{ABER}_{\xi}^{(\text {inter })}}{\gamma_{\mathbf{I}}^{-N_{r}}} \\
=4 N_{t}^{(\xi)}\left(\begin{array}{c}
2 N_{r}-1 \\
N_{r}
\end{array}\right) 2^{-\left(2 N_{r}+1\right)} \frac{2 N_{t}^{(\xi)}+2^{-N_{r}-1}-1}{2 N_{t}^{(\xi)}} \\
=\left(2 N_{t}^{(\xi)}+\left(2^{-N_{r}-1}-1\right)\right)\left(\begin{array}{c}
2 N_{r}-1 \\
N_{r}
\end{array}\right) 2^{-\left(2 N_{r}\right)} .
\end{aligned}
$$

Restating (37) for convenience, we have

$$
\begin{aligned}
\lim _{\gamma \rightarrow \infty} \frac{\mathrm{ABER}_{\mathrm{QAM}}}{\gamma^{-N_{r}}} \leq & \frac{4}{\log _{2}(\widetilde{M})}\left(1-\frac{1}{\sqrt{\widetilde{M}}}\right) \\
& \times\left(\frac{3}{2(\widetilde{M}-1)}\right)^{-N_{r}}\left(\begin{array}{c}
2 N_{r}-1 \\
N_{r}
\end{array}\right) 2^{-\left(2 N_{r}\right)} .
\end{aligned}
$$

Taking the ratio of (49) over (37) gives (38) as

$$
\lim _{\gamma \rightarrow \infty} \frac{\mathrm{ABER}_{\xi}^{(\text {inter })} / \gamma^{-N_{r}}}{\mathrm{ABER}_{\mathrm{QAM}} / \gamma^{-N_{r}}}=\frac{\left(2 N_{t}^{(\xi)}+2^{-N_{r}-1}-1\right)}{\left(\frac{2\left(4 N_{t}^{(\xi)}-1\right)}{3}\right)^{N_{r}} \frac{4}{\log _{2}\left(4 N_{t}^{(\xi)}\right)}\left(1-\frac{1}{\sqrt{4 N_{t}^{(\xi)}}}\right)}
$$

\section{Derivation of (43)}

$$
\begin{aligned}
& \operatorname{Pr}\{\mathbf{A} \neq \hat{\mathbf{A}}\}=\operatorname{Pr}\left\{\|\mathbf{y}-\mathbf{A}\|_{\mathrm{F}}^{2}>\|\mathbf{y}-\hat{\mathbf{A}}\|_{\mathrm{F}}^{2}\right\} \\
& =\operatorname{Pr}\left\{\sum_{r=1}^{N_{r}}\left[\left|y_{r}-A_{r}\right|^{2}\right]>\sum_{r=1}^{N_{r}}\left[\left|y_{r}-\hat{A}_{r}\right|^{2}\right]\right\} \\
& =\operatorname{Pr}\left\{\sum_{r=1}^{N_{r}}\left[2 \Re\left\{\left(\hat{A}_{r}-A_{r}\right) \eta^{*}\right\}\right]>\sum_{r=1}^{N_{r}}\left[\left|A_{r}-\hat{A}_{r}\right|^{2}\right]\right\} \\
& \Rightarrow \eta_{r}^{\text {new }} \sim \mathcal{N}\left(\sum_{r=1}^{N_{r}}\left[2 \Re\left\{\left(\hat{A}_{r}-A_{r}\right)^{*}\right\}\right], \sum_{r=1}^{N_{r}}\left[2 N_{o}\left|\hat{A}_{r}-A_{r}\right|^{2}\right]\right),
\end{aligned}
$$

where $\mathbf{A}=\frac{E_{m}}{2 N_{o}} \sum_{u=1}^{N_{u}} \alpha_{(u)} \mathbf{h}_{n_{t}^{(u)}} x^{(u)}$ and $\hat{\mathbf{A}}=\frac{E_{m}}{2 N_{o}}$ $\sum_{u=1}^{N_{u}} \alpha_{(u)} \mathbf{h}_{\hat{n}_{t}^{(u)}} \hat{x}^{(u)}$.

If we consider a Rayleigh fading channel, then we can derive the closed form solution for $\mathrm{E}_{\mathbf{H}}[\mathrm{PEP}(\cdot)]$ in (42) by using ([30], eq. 62). We note that by assuming a Rayleigh fading channel, the argument within (43) can be represented as the summation of $2 N_{r}$ squared Gaussian random variables, with zero mean and variance equal to 1 , which means that they can be described by a central Chi-squared distribution with $2 N_{r}$ degrees of freedom and a probability density function of

$$
\tilde{\rho}_{K}(\kappa)=\frac{1}{2^{N_{r}}\left(N_{r}-1\right) !} \kappa^{N_{r}-1} \exp (-\kappa / 2) .
$$

The result for $\mathrm{E}_{\mathbf{H}}[\mathrm{PEP}(\cdot)]$ is given as

$$
\mathrm{E}_{\mathbf{H}}[\mathrm{PEP}(\cdot)]=f(\tilde{\beta})^{N_{r}} \sum_{r=0}^{N_{r}-1}\left(\begin{array}{c}
N_{r}-1+r \\
r
\end{array}\right)(1-f(\tilde{\beta}))^{r}
$$

such that $f(\tilde{\beta})=\frac{1}{2}\left(1-\sqrt{\frac{\tilde{\beta}}{1+\tilde{\beta}}}\right)$ and $\tilde{\beta}=\frac{E_{m}}{4 N_{o}}$ $\sum_{u=1}^{N_{u}} \alpha_{(u)}^{2} \vartheta_{(u)}$

\section{Competing interests}

The authors declare that they have no competing interests.

\section{Acknowledgements}

We gratefully acknowledge the support from the Engineering and Physical Sciences Research Council (EP/G011788/1) in the United Kingdom for this study.

\section{Author details}

${ }^{1}$ Institute for Digital Communications, Joint Research Institute for Signal and Image Processing, School of Engineering, The University of Edinburgh, Edinburgh EH9 3JL, UK. ${ }^{2}$ Laboratory of Signals and Systems (L2S), French National Center for Scientific Research (CNRS), L'Ecole Supérieure d'Électricité (SUPÉLEC), University of Paris-Sud XI (UPS), 3 rue Joliot-Curie, 91192

Gif-sur-Yvette (Paris), France.

Received: 26 March 2012 Accepted: 4 September 2012

Published: 19 September 2012

\section{References}

1. J Mietzner, R Schober, L Lampe, WH Gerstacker, PA Hoeher, Multipleantenna techniques for wireless communications - a comprehensive literature survey. IEEE Commun. Surv. Tutor. 11(2), pp. 87-105 (2009)

2. R Mesleh, H Haas, Y Lee, S Yun, Interchannel interference avoidance in MIMO transmission by exploiting spatial information, in Proc. of the 16th IEEE International Symposium on Personal, Indoor and Mobile Radio Communications (PIMRC), vol. 1 ((Berlin Germany, 11-14 September 2005), pp. 141-145

3. R Mesleh, H Haas, S Sinanović, CW Ahn, S Yun, Spatial modulation. IEEE Trans. Veh. Technol. 57(4), pp. 2228-2241 (2008)

4. N Serafimovski, Di Renzo M, S Sinanović, RY Mesleh, H Haas, Fractional bit encoded spatial modulation (FBE-SM). IEEE Commun. Lett. 14(5), pp. 429-431 (2010)

5. A Younis, N Serafimovski, R Mesleh, H Haas, Generalized spatial modulation, in Asilomar Conference on Signals, Systems, and Computers (Pacific Grove,CA, USA, November 2010)

6. R Mesleh, M Di Renzo, H Haas, PM Grant, Trellis coded spatial modulation. IEEE Trans. Wirel. Commun. 9(7), pp. 2349-2361 (2010)

7. A Younis, R Mesleh, H Haas, PM Grant, Reduced complexity sphere decoder for spatial modulation detection receivers, in 2010 IEEE Global Telecommunications Conference (GLOBECOM 2010) (Miami, Florida, USA, December 2010), pp. 1-5

8. MD Renzo, H Haas, Performance analysis of spatial modulation, in International ICST Conference on Communications and Networking in China (CHINACOM) (Beijing, China, August 2010, pp. 1-7)

9. A Younis, Renzo M, R Mesleh, H Haas, Sphere decoding for spatial modulation, in Proc. of IEEE International Conference on Communications (IEEE ICC 2011) (Kyoto, Japan, 5-9 June 2011), pp. 1-6

10. J Jeganathan, A Ghrayeb, L Szczecinski, Spatial modulation: optimal detection and performance analysis. IEEE Commun. Lett. 12(8), pp. 545-547 (2008)

11. SU Hwang, S Jeon, S Lee, J Seo, Soft-output ML detector for spatial modulation OFDM systems. IEICE Electron Exp. 6(19), pp. 1426-1431 (2009)

12. M Di Renzo, H Haas, Spatial modulation with partial-CSI at the receiver: optimal detector and performance evaluation, in Proceedings of the 33rd 
IEEE conference on Sarnoff (IEEE Press, Piscataway, NJ, USA, 2010), pp. 58-63. http://portal.acm.org/citation.cfm?id=1843486.1843498

13. M Di Renzo, H Haas, Improving the performance of Ssace shift keying (SSK) modulation via opportunistic power allocation IEEE Commun. Lett. 14(6), pp. 500-502 (2010)

14. T Handte, A Muller, J Speidel, BER analysis and optimization of generalized spatial modulation in correlated fading channels, in Vehicular Technology Conference Fall (VTC Fall-2009) Anchorage Alaska, USA, IEEE, (September 2009), pp. 1-5

15. MD Renzo, H Haas, Bit error probability of SM-MIMO over generalized fading channels. IEEE Trans. Veh. Technol. 61(3), pp. 1124-1144 (2012)

16. E Basar, U Aygolu, E Panayirci, VH Poor, Space-time block coded spatial modulation. IEEE Trans. Commun. 59(3), pp. 823-832 (2011)

17. J Jeganathan, A Ghrayeb, L Szczecinski, A Ceron, Space shift keying modulation for MIMO channels. IEEE Trans. Wirel. Commun. 8(7), pp. 3692-3703 (2009)

18. JN Laneman, DNC Tse, GW Wornell, Cooperative diversity in wireless networks: efficient protocols and outage behavior. IEEE Trans. Inf. Theory. 50(12), pp. 3062-3080 (2004)

19. MO Hasna, M-S Alouini, End-to-end performance of transmission systems with relays over Rayleigh-fading channels. IEEE Trans. Wirel. Commun. 2(6), pp. 1126-1131 (2003)

20. N Serafimovski, S Sinanovic, M Di Renzo, H Haas, Dual-hop spatial modulation (Dh-SM), in Proc. of the Vehicular Technology Conference (VTC Spring), Budapest, Hungary, IEEE, 15-18, May 2011), pp. 1-5

21. M Di Renzo, H Haas, On the performance of SSK modulation over multipleaccess Rayleigh fading channels, in IEEE Global Telecommunications Conference (GLOBECOM) (Miami, Florida, USA, December 2010), pp. 1-6

22. M Di Renzo, H Haas, Bit error probability of space-shift keying MIMO over multiple-access independent fading channels. IEEE Trans. Veh. Technol. 60(8), pp. 3694-3711 (2011)

23. J Duplicy, B Badic, R Balraj, R Ghaffar, P Horvth, FKR Knopp, IZ Kovacs, HT Nguyen, D Tandur, G Vivier, MU-MIMO in LTE systems. EURASIP J. Wirel. Commun. Netw. 2011, p. 13 (2011)

24. JG Andrews, W Choi, RW Heath Jr, Overcoming interference in spatial multiplexing MIMO cellular networks. IEEE Wirel. Commun. Mag. 14(6), pp. 95-104 (2007)

25. D Gesbert, M Kountouris, RW Heath, C byoung Chae, T Salzer, From single user to multiuser communications: shifting the MIMO paradigm. IEEE Signal Process. Mag. 24, pp. 36-46 (2007)

26. CW Tan, AR Calderbank, Multiuser detection of alamouti signals. IEEE Trans. Commun. 57, pp. 2080-2089 (2009). http://dl.acm.org/citation. $\mathrm{cfm} ? \mathrm{id}=1651065.1651094$

27. G Auer, V Gianni, I Godor, P Skillermark, M Olsson, M Imran, M Gonzalez, C Desset, O Blume, A Fehske, How much energy is needed to run a wireless network? IEEE Wirel. Commun. 11, pp. 40-49 (2011)

28. C Desset, B Debaillie, V Giannini, A Fehske, G Auer, H Holtkamp, W Wajda, D Sabella, F Richter, MJ Gonzalez, H Klessig, I Godor, M Olsson, MA Imran, A Ambrosy, O Blume, Flexible power modeling of LTE base stations, in IEEE Wireless Communications and Networking Conference (WCNC), (Paris, France, April 2012), pp. 2858-2862

29. S Verdu, Multiuser Detection. (Cambridge University Press, Cambridge, MA), 1998)

30. M-S Alouini, A Goldsmith, A unified approach for calculating error rates of linearly modulated signals over generalized fading channels. IEEE Trans. Commun. 47(9), pp. 1324-1334 (1999)

31. M Di Renzo, H Haas, Bit error probability of spatial modulation (SM) MIMO over generalized fading channels. IEEE Trans Veh. Technol. 61, pp. 1124-1144 (2012)

32. MK Simon, M Alouini, Digital Communication over Fading Channels, 2nd edn (John Wiley \& Sons, Inc, NEw York, 2005). ISBN: 978-0-471-64953-3

33. S Verdu, Computational complexity of optimum multiuser detection. Algorithmica. 4, pp. 303-312 (1989). doi:10.1007/BF01553893

\section{doi:10.1186/1687-1499-2012-299}

Cite this article as: Serafimovski et al:: Multiple access spatial modulation. EURASIP Journal on Wireless Communications and Networking 2012 2012:299.

\section{Submit your manuscript to a SpringerOpen ${ }^{\mathcal{O}}$ journal and benefit from:}

- Convenient online submission

- Rigorous peer review

- Immediate publication on acceptance

- Open access: articles freely available online

- High visibility within the field

- Retaining the copyright to your article

Submit your next manuscript at $\gg$ springeropen.com 